\title{
Axial prestretch and circumferential distensibility in biomechanics of abdominal aorta
}

\author{
Lukáš Horný ${ }^{*}$, Marek Netušil ${ }^{2}$, Tereza Voňavková ${ }^{1}$ \\ ${ }^{1}$ Czech Technical University in Prague, Faculty of Mechanical Engineering, Technická 4, 166 07, \\ Prague, Czech Republic \\ ${ }^{2}$ Charles University in Prague, Faculty of Mathematics and Physics, Sokolovská 83, 186 75, Prague, \\ Czech Republic \\ *Corresponding author: lukas.horny @fs.cvut.cz, tel. +420 224352 690, fax +420 233322482
}

\begin{abstract}
Elastic arteries are significantly prestretched in an axial direction. This property minimises axial deformations during pressure cycle. Ageing-induced changes in arterial biomechanics, among others, are manifested via a marked decrease of the prestretch. Although this fact is well known, little attention has been paid to the effect of decreased prestretch on mechanical response. Our study presents the results of an analytical simulation of the inflation-extension behaviour of the human abdominal aorta treated as nonlinear, anisotropic, prestrained thin-walled as well as thick-walled tube with closed ends. The constitutive parameters and geometries for 17 aortas adopted from the literature were supplemented with initial axial prestretches obtained from the statistics of 365 autopsy measurements. For each aorta, the inflation-extension response was calculated three-times: with expected value of the initial prestretch and with the upper and lower confidence limit of the initial prestretch derived from the statistics. This approach enabled age-related trends to be evaluated bearing in mind the uncertainty in the prestretch. Despite significantly decreased longitudinal prestretch with age, the biomechanical response of human abdominal aorta changes substantially depending on the initial axial stretch used. In particular, substituting the upper limit of initial prestretch gave mechanical responses which can be characterised by (1) low variation in axial stretch, and (2) high circumferential distensibility during pressurisation, in contrast to the responses obtained for their weakly prestretched counterparts. The simulation also suggested the significant effect of the axial prestretch on the variation of axial stress in the pressure cycle. Finally, the obtained results are in accordance with the hypothesis that circumferential-to-axial stiffness ratio is the quantity relatively constant within this cycle.
\end{abstract}

Keywords: abdominal aorta; ageing; constitutive modelling; distensibility; prestretch; stiffness. 
Horny L, Netusil M, Vonavkova T (2014) Axial prestretch and circumferential distensibility in biomechanics of abdominal aorta. Biomechanics and Modeling in Mechanobiology 13(4):783-799. DOI: 10.1007/s10237-013-0534-8 MANUSCRIPT http://dx.doi.org/10.1007/s10237-013-0534-8

\section{Introduction}

There is extensive literature dealing with the circumferential behaviour of elastic arteries, e.g. aorta, carotids, iliacs (Dobrin 1978; Humphrey 2002; Shadwick 1999; Kalita and Schaefer 2008). This literature includes results of both ex vivo and in vivo approaches showing the unique Windkessel function of these arteries. The manner of how elastic arteries transmit pressure pulse wave has been linked to mortality (McEniery et al. 2007; Greeenwald 2007). Arteriosclerotic changes, which are responsible for age-related loss of elasticity (a key factor for pulse wave transmission), have subsequently been suggested as a potential target for cardiovascular therapy (O'Rourke and Hashimoto 2007). It was well established that circumferential distensibility declines with age. In contrast to the circumferential mechanical response, the axial behaviour of arteries in their natural (tubular) geometry has been studied less extensively, especially for human data which can only be found in a limited number of reports (Horny et al. 2013b).

Elastic arteries in situ are significantly prestretched in an axial direction (Dobrin and Doyle 1970; Han and Fung 1995; Learoyd and Taylor 1967). They retract upon excision and the difference between the in situ and ex situ length rapidly decreases in middle age and only small changes follow after the age of 60 (Horny et al. 2011, 2012a,b). For instance, a regression model adopted in this study from Horny et al. (2013a) respectively gives axial prestretch 1.33, 1.23, 1.08, and 1.05 at age 20,30, 60 and 70 , which implies a decrease of approx. $30 \%$ and $9.5 \%$ per decade with reference to 20 years of age (the supposed maximum prestretch due to the end of the growth period).

Axial prestress, induced by the prestretch, has an important physiological function. In an idealised case, it enables the artery to carry the pulse pressure with minimal variation in its length (SchulzeBauer et al. 2003; Sommer et al. 2010; Van Loon et al. 1977). It is, however, unknown how ageinginduced changes in prestress and stiffness are inter-related together. In other words, how the stress state of an artery is affected by a simultaneous decrease in the prestretch and an increase in the stiffness (at strains corresponding to in vivo loading). This interrelation is significantly complicated by the nonlinearity and anisotropy of arterial constitutive behaviour (Holzapfel et al. 2000; Holzapfel and Ogden 2010a).

Our study attempts to contribute to this topic with an analytical simulation of the inflation and extension behaviour of human abdominal aorta treated as a homogenous, nonlinear and anisotropic continuum. The constitutive model and its parameters are adopted from Labrosse et al. (2013) who have recently published the results of 17 inflation-extension tests with human abdominal aortas. Data describing the axial prestretch of aortas are adopted from Horny et al. (2013a). They systematically conducted autopsy measurements of the prestretch and their sample has reached a total of 365 observations which is suitable to be used as a representative of a population. 


\section{Methods}

\subsection{Constitutive model and parameters}

Although the biomechanics of large arteries has been extensively studied worldwide, scientific papers reporting the constitutive parameters obtained from pressurisation tests with human abdominal aorta are rare. Labrosse and co-workers have recently published data suitable for a purpose of computational simulation (Labrosse et al. 2013). They conducted inflation tests (with simultaneous free axial extension), determined constitutive parameters and discussed the results with reference to transmural stress distribution (residual strain/stress). Within our study, we adopted the constitutive model, material parameters and reference geometries (thickness, radius, opening angle) presented by them (Labrosse et al. 2013).

The constitutive model is based on the Fung-type exponential strain energy density function $W$ (1) which in the literature is referred to as Guccione's model (Guccione et al. 1991). $E_{R R}, E_{\Theta \Theta}$ and $E_{Z Z}$ respectively are the radial, circumferential and axial components of the Green-Lagrange strain tensor in the cylindrical coordinate system and $c_{0}, c_{1}, c_{2}$ are the material parameters.

$$
W=\frac{c_{0}}{2}\left(e^{c_{1} E_{\Theta \Theta}^{2}+c_{2}\left(E_{Z Z}^{2}+E_{R R}^{2}\right)}-1\right)
$$

The artery wall was considered to be incompressible. Stress-strain relationship is then obtained in the form of (2). Here $\mathbf{F}$ denotes the deformation gradient, $\mathbf{E}$ denotes the Green-Lagrange strain tensor defined as $\mathrm{E}=1 / 2\left(\mathbf{F}^{\mathrm{T}} \mathbf{F}-\mathbf{I}\right), p$ is hydrostatic stress resulting from incompressibility constraint, $\mathbf{I}$ denotes second order unit tensor, and $\sigma$ is the Cauchy stress tensor.

$$
\sigma=\mathbf{F} \frac{\partial W}{\partial \mathbf{E}} \mathbf{F}^{T}-\mathbf{I} p
$$

Material parameters, age (38-77 years) and gender are specified in Table 1.

\subsection{Axial prestretch $\lambda_{z} z^{i n i}$}

Axial prestretch of the large arteries cannot be directly measured in the living due to the destructive nature of such an experiment (a segment of an artery has to be excised from a body). Since Labrosse et al. (2013) did not report specific values of the prestretch, we adopted population data from Horny et al. (2013a). Horny et al. (2013a) measured the retraction of segments of the human abdominal aorta in 365 regular autopsies. The data sample of 365 measurements is large enough to capture trends and variability in the prestretch occurring in the population. The data has been fitted to a regression equation (3) describing dependence on age ( $a, b$ denotes regression parameters and $x$ denotes age [years]).

$$
\lambda_{z Z}^{i n i}=a x^{b}
$$

In the following simulations, initial axial prestretch $\lambda_{z Z}{ }^{i n i}$ will be prescribed to the value obtained from (3) after the substitution of specific age (Table 1). Since no measurement is free from uncertainty and population data is used, the simulations will also employ the upper and lower limit of the 
Horny L, Netusil M, Vonavkova T (2014) Axial prestretch and circumferential distensibility in biomechanics of abdominal aorta. Biomechanics and Modeling in Mechanobiology 13(4):783-799. DOI: 10.1007/s10237-013-0534-8 MANUSCRIPT http://dx.doi.org/10.1007/s10237-013-0534-8

prediction interval of the prestretch. Specifically, a 95\%-confidence interval for a prediction given by the regression model. This approach will enable us to evaluate the expected behaviour of abdominal aorta (expected behaviour corresponds to estimates based on eq. 3) and its limits implicated by variance in the initial prestretch. They will be denoted $U L$ (upper limit) and $L L$ (lower limit) and are based on the classical linear regression model and its logarithmic transformation (4). Here $y$ denotes initial prestretch, $x$ denotes age, $x_{i}$ denotes $i$-th observed age, $S_{e}$ is residual standard deviation and $t$ is a quantile of Student- $t$ distribution for $m$ degrees of freedom at significance $\alpha$. The significance level 0.05 is used within all the study. We note that assumptions of classical linear model have been proven in Horny et al. (2012b).

$$
\ln y=\ln a+b \ln x \pm t_{\frac{\alpha}{2}}(m) S_{e} \sqrt{1+\frac{1}{n}+\frac{\left(\ln x-\operatorname{Mean}\left(\ln x_{i}\right)\right)^{2}}{\sum_{i=1}^{n}\left(\ln x_{i}-\operatorname{Mean}\left(\ln x_{i}\right)\right)^{2}}}
$$

\subsection{Computational model for inflation-extension response}

Herein we will focus on the quasi-static problem because it is the most frequently used in constitutive model determination. The artery wall will be considered as a one-layered, incompressible, nonlinear, anisotropic, and closed tube which is initially prestrained to its in situ length and is free at its outer deformed radius $r_{o}$ and distended by internal pressure $P$ at inner radius $r_{i}$. With regard to the thickness of the artery wall, we employed both thin-walled and thick-walled approaches. This is done for two basic reasons. First, the thin-walled model, which operates with mean wall stresses acting at middle radius $r_{m}$, may be regarded as more suitable when results of the simulation are compared with in vivo data obtained by ultrasound methods because there may be a problem in identifying the outer radius of the wall, in contrast to media-adventitia interface (this interface could be used as an estimate of $r_{m}$ ). On the other hand, the thin-walled model (in contrast to thick-walled) cannot capture residual strain/stress which may significantly change the true stress/strain state of the material.

Thin-walled model. In the thin-walled approximation, the equilibrium equations are written in the form of (5) with kinematic equations (6).

$$
\begin{array}{r}
\sigma_{r r}=-\frac{P}{2} \quad \sigma_{\theta \theta}=\frac{r_{m} P}{h} \quad \sigma_{z z}=\frac{F_{r e d}}{2 \pi r_{m} h}+\frac{r_{m} P}{2 h} \\
h=\lambda_{r R} H \quad r_{m}=\lambda_{\theta \Theta} R_{m} \quad z=\lambda_{z Z} Z
\end{array}
$$

In (5) $\sigma_{r r}, \sigma_{\theta \theta}$, and $\sigma_{z z}$ respectively denote mean radial, circumferential and axial Cauchy stress at middle radius $r_{m}=\left(r_{i}+r_{o}\right) / 2$, and $h$ is the thickness in the deformed state. Middle reference radius is denoted $R_{m}\left(R_{m}=R_{i}+H / 2\right)$, reference inner radius $R_{i}$, and $H$ denotes reference thickness (see Table 1 for specific values). From (6) the deformation gradient can be written as $\mathbf{F}=\operatorname{diag}\left[\lambda_{r R}, \lambda_{\theta \Theta}, \lambda_{z z}\right]$. 
Horny L, Netusil M, Vonavkova T (2014) Axial prestretch and circumferential distensibility in biomechanics of abdominal aorta. Biomechanics and Modeling in Mechanobiology 13(4):783-799. DOI: 10.1007/s10237-013-0534-8 MANUSCRIPT http://dx.doi.org/10.1007/s10237-013-0534-8

$F_{\text {red }}$ in $(5 \mathrm{c})$ is external axial force necessary to obtain the in situ length corresponding to $\lambda_{z z^{i n i}}$ measured during autopsy. In ex vivo experiments, it is frequently generated with a hanging mass connected to a specimen (vertical configuration of the inflation-extension test). The denotation Fred was chosen with respect to nomenclature used in Holzapfel et al. (2000), Holzapfel and Ogden (2010a), and Ogden and Saccomandi (2007). This force is developed during the growth period and the literature suggests that elastin fibres are responsible for bearing this load (Carta et al. 2009; Humphrey et al. 2009). The key problem is that we in fact do not know how large in vivo $F_{\text {red }}$ is. We only have evidence that arteries retract upon excision. Statistics of $\lambda_{z} z^{i n i}$ are thus obtained, since measured during autopsy, at $P=0$. This motivated us to employ $F_{\text {red }}$ as a constant during the pressurisation. Throughout the pressurisation, the mechanical response of the artery has to satisfy equilibrium equations (5) and simultaneously mechanical state of the material has to conform to the constitutive equations (2). Combining (2) and (5) and interchanging variables in $W$ to the deformation gradient, the system (7) is obtained.

$$
\lambda_{r R} \frac{\partial W}{\partial \lambda_{r R}}-p=-\frac{P}{2} \quad \lambda_{\theta \Theta} \frac{\partial W}{\partial \lambda_{\theta \Theta}}-p=\frac{r_{m} P}{h} \quad \lambda_{z Z} \frac{\partial W}{\partial \lambda_{z Z}}-p=\frac{F_{r e d}}{2 \pi r_{m} h}+\frac{r_{m} P}{2 h}
$$

We would like to note explicitly that in the chosen approach $\lambda_{z} z^{i n i}$ is the constant used to compute Fred, but does not necessarily correspond to $\lambda_{z z}$ which can change during pressurisation (according to the system 7). This approach respects the fact the in vivo axial stretch has been proven to be slightly different from the prestretch measured in autopsy (Humphrey et al. 2009).

The system (7) describes the inflation-extension of the initially prestrained artery and was solved in the following steps:

1. Specific donor is chosen from Table $1 \rightarrow R_{i}, H, c_{0}, c_{1}, c_{2}$, and age.

2. From (3) expected $\lambda_{z} z^{i n i}$ is estimated.

3. Derivatives at left-hand side in (7) are conducted. Subsequently $p$ is eliminated from (7b) and (7c) using (7a). In the remaining system, (7b) and (7c), $\lambda_{r R}$ is substituted with $1 /\left(\lambda_{\theta \Theta} \cdot \lambda_{z z}\right)$ because for incompressible material $\operatorname{det} \mathbf{F}=1$ holds.

4. Prestretching force $F_{\text {red }}$ corresponding to $\lambda_{z} z^{\text {ini }}$ is computed from (7b) and (7c) at $P=0$ (both equations (7b) and (7c) are necessary because one also has to determine $\lambda_{\theta \Theta^{\text {jni }}}$ corresponding to prestretched but unpressurised tube).

5. The system (7b) and (7c) is now numerically solved for unknown $\lambda_{\theta \Theta}$ and $\lambda_{z z}$ (with $F_{\text {red }}$ substituted from step 4$)$ at $P=1,2, . ., 20 \mathrm{kPa}$.

6. When $\lambda_{\theta \Theta}$ and $\lambda_{z z}$ are determined, $\sigma_{r r}, \sigma_{\theta \theta}$, and $\sigma_{z z}$ can be calculated substituting the results into (5).

7. Instead of (3) the equation (4) is used to compute $\lambda_{z Z, u L L^{i n i}}\left(\lambda_{z Z, L L}{ }^{i n i}\right)$ and steps 3. - 6. are repeated to obtain results for upper limit (lower limit) of initially prestretched arteries. 
Thick-walled model. Elastic arteries are residually stressed in their unloaded configuration (Rachev and Greenwald 2003). The basic approach incorporating this fact into the computational model is to consider the opened up configuration as the reference one. When the unloaded ring of an artery is cut radially, it springs to an opened configuration which is (in the first approximation) considered to be stress-free and the geometry is modelled as a circular sector with inner radius $\rho_{i}$, outer radius $\rho_{o}$ and sector angle $2 \psi$. The so-called opening angle (inscribed angle in the sector), frequently used to characterise residual strain, is then given as $\pi-\psi$.

Equilibrium equations, with substituted constitutive model, describing the response of the closed thick-walled tube to internal pressure and prestretching force can be written in the form of (8). They are adopted from Labrosse et al. (2013). A detailed derivation can be found in Matsumoto and Hayashi (1996).

$$
P=\int_{r_{i}}^{r_{0}} \lambda_{\theta \Theta} \frac{\partial \hat{W}}{\partial \lambda_{\theta \Theta}} \frac{d r}{r} \quad F_{r e d}=\pi \int_{r_{i}}^{r_{o}}\left(2 \lambda_{z Z} \frac{\partial \hat{W}}{\partial \lambda_{z Z}}-\lambda_{\theta \Theta} \frac{\partial \hat{W}}{\partial \lambda_{\theta \Theta}}\right) r d r
$$

Here $\hat{W}$ is the strain energy density (1) with variables changed to the components of $\mathbf{F}$ and $\lambda_{r R}$ substituted by $\lambda_{r R}=1 /\left(\lambda_{\theta \Theta} \lambda_{z z}\right)$. Circumferential stretch $\lambda_{\theta \Theta}$ is considered to be a function of the deformed radius $r\left(r_{i} \leq r \leq r_{0}\right)$ and is expressed with respect to the radius in opened configuration $\rho$ ( $\left.\rho_{i} \leq \rho \leq \rho_{o}\right), \lambda_{\theta \Theta}=\pi r /(\psi \rho)$. Axial stretch $\lambda_{z z}$ is considered to be uniform along the length and thickness of the tube $\left(\lambda_{z z}=\right.$ constant). The equations (8) presume that boundary conditions $\sigma_{r r}\left(r_{o}\right)=0$ and $\sigma_{r r}\left(r_{i}\right)=-P$ are applied. The system (8) was used to simulate the inflation-extension response of aortas in the following way:

1. Specific donor is chosen from Table $1 \rightarrow R_{i}, H, c_{0}, c_{1}, c_{2}$, opening angle and age.

2. From (3) expected $\lambda_{z} z^{i n i}$ is estimated.

3. Integrands in (8) are expressed as functions of $r$ and $\lambda_{z z} . r_{o}=r_{o}\left(r_{i}\right)$ is used in upper bounds of integrals (from incompressibility condition).

4. Prestretching force $F_{\text {red }}$ corresponding to $\lambda_{z} z^{\text {ini }}$ is computed from the system (8) at $P=0$ (both equations (8a) and (8b) are necessary because one also has to determine $r_{i}^{\text {ini }}$ corresponding to residually stressed and axially prestretched but unpressurised tube).

5. The system (8) is now numerically solved for unknown $r_{i}$ and $\lambda_{z z}$ (with $F_{\text {red }}$ substituted from step 4) at $P=1,2, . ., 20 \mathrm{kPa}$.

6. When $r_{i}$ and $\lambda_{z z}$ are found, $\sigma_{r r}(r), \sigma_{\theta \theta}(r)$, and $\sigma_{z z}(r)$ can be calculated from equations (9) considering that $\lambda_{\theta \Theta}=\pi r /(\psi \rho)$.

$$
\sigma_{r r}=-\int_{r}^{r_{\theta}} \lambda_{\theta \Theta} \frac{\partial \hat{W}}{\partial \lambda_{\theta \Theta}} \frac{d x}{x} \quad \sigma_{\theta \theta}=\lambda_{\theta \Theta} \frac{\partial \hat{W}}{\partial \lambda_{\theta \Theta}}+\sigma_{r r} \quad \sigma_{z z}=\lambda_{z Z} \frac{\partial \hat{W}}{\partial \lambda_{z Z}}+\sigma_{r r}
$$

A detailed derivation of (9) can be found in Matsumoto and Hayashi (1996). It is important to emphasize that in (9a) the right-hand expression is a function of the lower bound $r$.

6. Instead of (3), the equation (4) is used to compute $\lambda_{z Z, u L^{i n i}}\left(\lambda_{z Z, L L} L^{i n i}\right)$ and steps 4. - 5. are repeated to obtain results for upper limit (lower limit) of initially stretched arteries. 
Horny L, Netusil M, Vonavkova T (2014) Axial prestretch and circumferential distensibility in biomechanics of abdominal aorta. Biomechanics and Modeling in Mechanobiology 13(4):783-799. DOI: 10.1007/s10237-013-0534-8 MANUSCRIPT http://dx.doi.org/10.1007/s10237-013-0534-8

Using the procedures described above, $\lambda_{\theta \Theta}(P)$ and $\lambda_{z}(P)$ in the thin-walled, and $\lambda_{\theta \Theta}(r, P)$ and $\lambda_{z z}(P)$ in the thick-walled approach were determined for all involved donors numerically in Maple 16. The results were used to compute variations in the stretches during the pressure cycle $\mathcal{E}_{i I}=\lambda_{i I}\left(P_{S Y S}\right)-\lambda_{i I}\left(P_{D I A}\right)$ for $i I=\theta \Theta$ and $z Z$, to create $P-\lambda_{\theta \Theta}$ and $P-\lambda_{z Z}$ dependences, and to quantify changes of the axial stress in the course of the pressurisation. Changes of the axial stress were quantified as relative increments between diastole and systole $\left[\sigma_{z z}\left(P_{S Y S}\right)-\sigma_{z z}\left(P_{D I A}\right)\right] / \sigma_{z z}\left(P_{D I A}\right)$.

In what follows, the results of the thick-walled model and thin-walled model will not be distinguished by special symbols for quantities in hand but they always will be distinguished by the radius at which they were obtained. This indicates that the results computed at $r_{i}$ and $r_{o}$ are always given by the thick-walled model with incorporated residual strain and the results computed with the thin-walled model are always related to $r_{m}$.

\subsection{Stiffness}

Chen et al. (2008) have suggested incorporating the assumption of the constant ratio between circumferential and longitudinal elastic modulus of the artery wall during the pressure cycle to overcome the impossibility of measuring axial stress in the constitutive parameter identification procedure conducted with living subjects. To evaluate this hypothesis, components of the elasticity tensor C (tensor of elastic module) in the material description have been computed. The material description was used to hold consistency with the definition of the strain energy density (1) defined also in the material description.

C is defined as the derivative (10) of the second Piola-Kirchhoff stress tensor $\mathbf{S}$ with respect to the Green-Lagrange strain tensor E, Holzapfel (2000) ch. 6.6.

$$
\mathbf{C}=\frac{\partial S}{\partial E}
$$

The Second Piola-Kirchhoff stress tensor $\mathbf{S}$ measures the stress state of a body using material description. One can say that $\mathbf{S}$ is defined with respect to the material (undeformed) configuration. This is in contrast to the Cauchy stress tensor $\sigma$ which measures the stress state of a body in the deformed configuration (spatial description). Both stress tensors can be mutually transformed using (11) because the deformation gradient $\mathbf{F}$ creates a map from an undeformed to a deformed configuration. The equation (11) involves the inverse of $\mathbf{F}$ since transformation proceeds in the opposite direction - from deformed to undeformed configuration.

$$
\mathbf{S}=J \mathbf{F}^{-1} \sigma \mathbf{F}^{-T}
$$

$J$ in (11) denotes the ratio between volume of a body in the deformed and undeformed configuration and, in our specific case, for the incompressible material $J=1$. Since in our study only diagonal components of second order tensors are involved, the equation (11) reduces to $S_{K K}=\lambda_{k K}{ }^{-2} \sigma_{k k}$.

Thin-walled model. $C_{\Theta \Theta \Theta \Theta}$ and Czzzz were computed from (10) with the substituted material counterpart of (2) which can be written in the form of (12).

$$
\mathbf{S}=\frac{\partial W}{\partial \mathbf{E}}-p(2 \mathbf{E}+\mathbf{I})^{-1}
$$


Specific expression for $p$ is obtained from the radial component of the equation (12). Considering (11), $S_{R R}=\lambda_{r R}{ }^{-2} \sigma_{r r}$ holds and $p$ can be written as $p=\left(2 E_{R R}+1\right) \cdot \partial W / \partial E_{R R}-\sigma_{r r}$. In the case of the thin-walled model, $-P / 2$ is substituted into the radial Cauchy stress $\sigma_{r r}$.

Thick-walled model. In the thick-walled model, the situation is somewhat more complicated. We will use equations (9b) and (9c) with substituted (9a). They will be transformed into the components of $\mathbf{S}$. This results in (13) and (14).

$$
\begin{aligned}
S_{\Theta \Theta} & =\frac{\partial \hat{W}}{\partial E_{\Theta \Theta}}-\left(2 E_{\Theta \Theta}+1\right)^{-1} \int_{r}^{r_{r}}\left(2 E_{\Theta \Theta}+1\right) \frac{\partial \hat{W}}{\partial E_{\Theta \Theta}} \frac{d x}{x} \\
S_{Z Z} & =\frac{\partial \hat{W}}{\partial E_{Z Z}}-\left(2 E_{Z Z}+1\right)^{-1} \int_{r}^{r_{o}}\left(2 E_{\Theta \Theta}+1\right) \frac{\partial \hat{W}}{\partial E_{\Theta \Theta}} \frac{d x}{x}
\end{aligned}
$$

Using the product rule for differentiation, equations (13) and (14) give (15) and (16), respectively.

$$
\begin{aligned}
C_{\Theta \Theta \Theta \Theta}= & \frac{\partial S_{\Theta \Theta}}{\partial E_{\Theta \Theta}}=\frac{\partial^{2} \hat{W}}{\partial E_{\Theta \Theta}^{2}}+2\left(2 E_{\Theta \Theta}+1\right)^{-2} \int_{r}^{r_{o}}\left(2 E_{\Theta \Theta}+1\right) \frac{\partial \hat{W}}{\partial E_{\Theta \Theta}} \frac{d x}{x} \\
& -\left(2 E_{\Theta \Theta}+1\right)^{-1} \frac{\partial}{\partial E_{\Theta \Theta}} \int_{r}^{r_{o}}\left(2 E_{\Theta \Theta}+1\right) \frac{\partial \hat{W}}{\partial E_{\Theta \Theta}} \frac{d x}{x} \\
C_{Z Z Z Z}= & \frac{\partial S_{Z Z}}{\partial E_{Z Z}}=\frac{\partial^{2} \hat{W}}{\partial E_{Z Z}^{2}}+2\left(2 E_{Z Z}+1\right)^{-2} \int_{r}^{r_{o}}\left(2 E_{\Theta \Theta}+1\right) \frac{\partial \hat{W}}{\partial E_{\Theta \Theta}} \frac{d x}{x} \\
& -\left(2 E_{Z Z}+1\right)^{-1} \frac{\partial}{\partial E_{Z Z}} \int_{r}^{r_{o}}\left(2 E_{\Theta \Theta}+1\right) \frac{\partial \hat{W}}{\partial E_{\Theta \Theta}} \frac{d x}{x}
\end{aligned}
$$

To compute derivatives of the integrals in (15) and (16), the chain rule in the form $\partial(-) / \partial E_{K K}=[\partial(-) / \partial r] \cdot\left[\partial r / \partial E_{K K}\right](K=\Theta$ and $Z)$ is adopted. Since the differentiation is conducted with respect to $r$ and $r$ simultaneously is a variable integration bound (the integrals are understood as the functions of the lower bound), the procedure leads to equations (17) and (18). Note that $d\left(\int_{r} r f(x) d x\right) / d r=-f(r)$.

$$
\begin{gathered}
C_{\Theta \Theta \Theta \Theta}=\frac{\partial S_{\Theta \Theta}}{\partial E_{\Theta \Theta}}=\frac{\partial^{2} \hat{W}}{\partial E_{\Theta \Theta}^{2}}+2\left(2 E_{\Theta \Theta}+1\right)^{-2} \int_{r}^{r_{o}}\left(2 E_{\Theta \Theta}+1\right) \frac{\partial \hat{W}}{\partial E_{\Theta \Theta}} \frac{d x}{x}+\frac{\partial \hat{W}}{\partial E_{\Theta \Theta}} \frac{1}{r} \frac{\partial r}{\partial E_{\Theta \Theta}} \\
C_{Z Z Z Z}=\frac{\partial S_{Z Z}}{\partial E_{Z Z}}=\frac{\partial^{2} \hat{W}}{\partial E_{Z Z}^{2}}+2\left(2 E_{Z Z}+1\right)^{-2} \int_{r}^{r_{o}}\left(2 E_{\Theta \Theta}+1\right) \frac{\partial \hat{W}}{\partial E_{\Theta \Theta}} \frac{d x}{x}+\frac{2 E_{\Theta \Theta}+1}{2 E_{Z Z}+1} \frac{\partial \hat{W}}{\partial E_{\Theta \Theta}} \frac{1}{r} \frac{\partial r}{\partial E_{Z Z}}
\end{gathered}
$$

What remains to be clarified are derivatives $\partial r / \partial E_{\Theta \Theta}$ and $\partial r / \partial E_{z z}$. The expression $r=r\left(E_{\Theta \Theta}, E_{Z Z}\right)$ can be obtained considering a volume preservation during the deformation: $\pi\left(r_{\mathrm{o}}^{2}-r^{2}\right)=\psi L\left(\rho_{\mathrm{o}}^{2}-\rho^{2}\right)$. This relation equals the volume of elongated (to length $l$ ) and pressurized tube limited by outer deformed radius $r_{o}$ and variable radius $r$ to the volume of the stress-free (opened up) cylindrical sector (of the length $L$ ) limited by outer radius $\rho_{o}$ and variable radius $\rho$. Considering $\lambda_{z} z$ $=l / L$, one obtains (19) substituting $\rho$ from above mentioned volume preservation into $\lambda_{\theta \Theta}=\pi r /(\psi \rho)$. 


$$
\lambda_{\theta \Theta}=\frac{\pi r}{\psi \sqrt{\rho_{o}^{2}-\frac{\pi}{\psi} \lambda_{z Z}\left(r_{o}^{2}-r^{2}\right)}}
$$

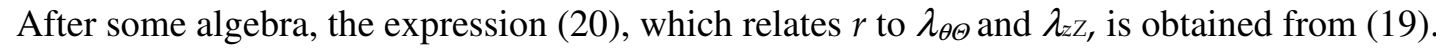

$$
r=\sqrt{\frac{\lambda_{\theta \Theta}^{2} \psi\left(\psi \rho_{o}^{2}-\pi r_{o}^{2} \lambda_{z Z}\right)}{\pi\left(1-\lambda_{\theta \Theta}^{2} \psi \lambda_{z Z}\right)}}
$$

One can arrive to the final expression $r=r\left(E_{\Theta \Theta}, E_{\mathrm{ZZ}}\right)$ substituting stretches with Green-Lagrange strain components, $\lambda_{\theta \Theta}=\left(2 E_{\Theta \Theta}+1\right)^{1 / 2}$ and $\lambda_{z z}=(2 E z z+1)^{1 / 2}$. Finally, the stiffness ratio $C_{\Theta \Theta \Theta \Theta} / C_{z z z z}$ and its relative increment between diastole and systole (normalised with respect to diastole) can be computed.

\subsection{Blood pressure in ageing}

It is well known that the character of pressure pulses in human arteries changes with age (Greenwald 2007; O'Rourke and Hashimoto 2007). With regard to this fact, we adopted numerical values of the diastolic and systolic pressure from recent epidemiological study conducted by Wilkins et al. (2010). They evaluated results of Canadian Health Measures Survey 2007 - 2009. Specific values used in our study are listed in Table 2. They were obtained as linear interpolation (with respect to variable age) of the data found in Figure 1 in Wilkins et al. (2010). The data in the original source is gender specific and represents the average in the population. That is, the averaged value is obtained considering healthy, successfully treated, unsuccessfully treated and untreated hypertensive/hypotensive population in the given ageing period.

\subsection{Correlation}

The linear correlation coefficient $R$ was computed for all treated quantities to obtain a basic estimate of their dependence on age. It is supplemented with the test of the hypothesis $R=0$ (against alternative $R \neq 0$ ) based on the statistics $T=R\left[(n-2) /\left(1-R^{2}\right)\right]^{1 / 2}$ which was evaluated by $p$-value (here $n$ is the number of observations). Results were considered to be statistically significant at the level 0.05 within this study.

\section{Results}

In the present simulation, we would like to show how the mechanical response of human abdominal aorta changes because of both changed constitutive parameters and decreased longitudinal prestretch. Details of involved donors are listed in Table 1 (geometry and material parameters) and in Table 2 (estimated prestretch and diastolic and systolic pressure). 
Horny L, Netusil M, Vonavkova T (2014) Axial prestretch and circumferential distensibility in biomechanics of abdominal aorta. Biomechanics and Modeling in Mechanobiology 13(4):783-799. DOI: 10.1007/s10237-013-0534-8 MANUSCRIPT http://dx.doi.org/10.1007/s10237-013-0534-8

\subsection{Initial axial prestretch}

Data describing initial axial prestretch was collected during autopsies as described in Horny et al. (2013a). The regression equation (3) was fitted to the resulting sample with parameters $a=2.4016$ [1/year]; $b=-0.1957$ [-]. Regression curve and data are depicted in Figure 1. Limits for interval of $95 \%$-confidence of a prediction (an interval into which a future observation will fall with a probability equal to 0.95 ) are also depicted. Within the text, these limits are denoted $\lambda_{z Z \text {, uL }}{ }^{\text {ini }}$ (upper) and $\lambda_{z Z, L L}{ }^{i n i}$ (lower). Linear correlation coefficient for $\ln \left(\lambda_{z z^{i n i}}\right)-\ln (A g e) \quad R=-0.903$ ( $p$-value $\left.<0.001\right)$ confirmed a strong correlation between age and axial prestretch. Specific values for $\lambda_{z} z^{i n i}, \lambda_{z} z, u L^{i n i}$, and $\lambda_{z Z, L L^{i n i}}$ used in the simulations are listed in Table 2.

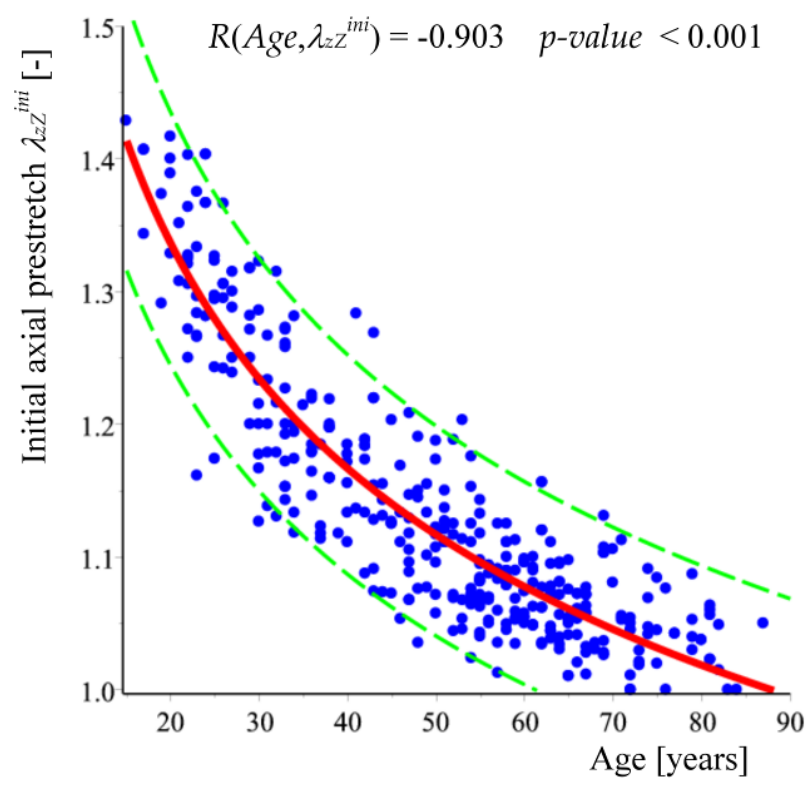

Regression model - expected value Observation 95\%-Prediction interval upper limit $\lambda_{z Z, u L}{ }^{i n i}$ and lower limit $\lambda_{z Z, L L}{ }^{i n i}$
Figure 1. Dependence of initial axial prestretch (found in autopsy) on age. Regression model for expected value $\lambda z z^{i n i}$ - thick red curve; upper limit $\lambda_{z Z, U L^{i n i}}$ and lower limit $\lambda_{z Z, L L}{ }^{i n i}$ of $95 \%$-prediction interval - green dashed curves; observations - blue points. Estimated parameters for regression equation (3) are $a=2.4016$ [1/year]; $b=-0.1957$ [-]. The data was adopted from Horny et al. (2013a). Since the lower limit of the prediction interval approaches 1 at the age of 61 years (no axial prestretch) and governed by (4) follows with values smaller than 1 (i.e. axial precompresion), it was decided to prescribe $\lambda_{z Z, L L}$ ini $=1$ for age $>61$ years. This was motivated by two facts: (1) Horny et al. (2013a) did not report any precompressed artery in their sample, (2) it is not clear whether the constitutive equations used in this study are suitable to describe precompressed arteries.

The prediction interval for $\lambda_{z} z^{i n i}$ based on (4) gives the lower limit smaller than 1 for age $>61$ (Figure 1). This is the consequence of the used methodology (expectation \pm uncertainty given as a function of a variance). Nevertheless, Horny et al. (2013a) did not report any abdominal aorta with $\lambda_{z} z^{i n i}<1$ (i.e. precompressed artery). Considering this fact in what follows, we have decided to substitute the exact results of the equation (4) with $\lambda_{z Z, L L}$ ini $=1$ for donors with age higher than 61 . 
Horny L, Netusil M, Vonavkova T (2014) Axial prestretch and circumferential distensibility in biomechanics of abdominal aorta. Biomechanics and Modeling in Mechanobiology 13(4):783-799. DOI: 10.1007/s10237-013-0534-8 MANUSCRIPT http://dx.doi.org/10.1007/s10237-013-0534-8

Table 1. Age, gender, geometry and constitutive parameters of involved donors; adopted from Labrosse et al. (2013).

\begin{tabular}{|c|c|c|c|c|c|c|c|c|c|c|c|c|c|c|c|c|c|}
\hline ID+ & F49 & F50 & F63 & F65 & M38 & M42 & M57 & M60 & M61a & M61b & M66 & M67a & M67b & M70a & M70b & M71 & M77 \\
\hline $\begin{array}{l}\text { Opening } \\
\text { angle }\left[{ }^{\circ}\right]\end{array}$ & 252 & 323 & 96 & 248 & 117 & 125 & 322 & 156 & 270 & 335 & 253 & 118 & 174 & 208 & 201 & 118 & 135 \\
\hline $\begin{array}{c}\boldsymbol{R}_{i} \\
{[\mathrm{~mm}]}\end{array}$ & 5.9 & 6.7 & 5.4 & 6.2 & 5.3 & 6.5 & 7.5 & 6.3 & 7.7 & 7.3 & 7.2 & 8 & 7.9 & 7.1 & 7.4 & 10 & 7 \\
\hline $\begin{array}{c}H \\
{[\mathrm{~mm}]}\end{array}$ & 1.51 & 1.14 & 0.96 & 1.21 & 1.22 & 1.56 & 1.28 & 1.69 & 1.22 & 1.62 & 1.78 & 1.58 & 1.26 & 1.23 & 1.64 & 1.72 & 1.5 \\
\hline $\begin{array}{c}c_{0} \\
{[\mathrm{kPa}]}\end{array}$ & 8.4 & 8.4 & 23 & 1.6 & 14.7 & 41.8 & 0.8 & 7.6 & 2.4 & 2.3 & 9.4 & 3.5 & 2.2 & 14 & 1.8 & 17 & 1.2 \\
\hline $\begin{array}{c}c 1 \\
{[-]}\end{array}$ & 5.09 & 15.21 & 4.07 & 9.26 & 3.04 & 1.54 & 6.74 & 2.96 & 37.53 & 6.82 & 7.81 & 24.47 & 56.69 & 16.09 & 18.62 & 13 & 41.08 \\
\hline $\begin{array}{l}c^{2} \\
{[-]}\end{array}$ & 8.18 & 9.67 & 7.2 & 11.77 & 7.38 & 1.44 & 12.44 & 10.23 & 34.01 & 19.16 & 12.5 & 27.9 & 41.66 & 7.38 & 35.99 & 11.85 & 49.51 \\
\hline
\end{tabular}

$+I D$ indicates sex (female/male) and age [years] of donors.

\subsection{Inflation-extension response}

Prescribed referential geometry, initial prestretch and constitutive parameters (Table 1 and 2) enabled the systems (7) and (8) to be solved with respect to $\lambda_{\theta \Theta}$ and $\lambda_{z z}$ for defined internal pressure $P$. Two representatives of $P-\lambda_{\theta \Theta}$ and $P-\lambda_{z Z}$ are shown in Figure 2 (M38) and Figure 3 (F65). For the sake of comparison, the results of the thick-walled model with incorporated residual strain as well as the results of the thin-walled model are depicted. Changes in the inflation characteristic induced by the prestretch are demonstrated for $\lambda_{z} z^{i n i}=1.0,1.1,1.2,1.3,1.4$ with $P=0-20 \mathrm{kPa}$. The higher the axial prestretch, the lower the initial circumferential stretch is. It is clearly evident that axial deformation at physiological pressures $(10-16 \mathrm{kPa})$ depends on initial prestretch; a property of the so-called inversion point is exhibited. The inversion point is the value of axial prestretch in the $P-\lambda_{z z}$ diagram which, in an idealised case, divides the diagram into inflation-extension and inflation-shortening behaviour (Ogden and Saccomandi 2007; Schulze-Bauer et al. 2003). Considering $P-\lambda_{\theta \Theta}$, an increased axial prestretch induces a left-side shift as expected. However, it also makes an inflexion point on $P-$ $\lambda_{\theta \Theta}$ more discernible and decreases the steepness of the curve at physiological pressures.

The effect of the initial axial prestretch modelled by (3) and (4) is shown in detail in Figure 4 for M61 (computed with thin-walled model). The upper panel shows the $P-\lambda_{z z}$ curve and lower panel $P-$ $\lambda_{\theta \Theta}$. Expected behaviour $\left(\lambda_{z} z^{i n i}\right.$ corresponding just to the regression equation (3)) is depicted with a blue solid curve laying in between curves computed with $\lambda_{z Z, L L}$ ini (black dots) and $\lambda_{z Z, u L}$ ini (red dashes) which are based on (4). It is clearly noticeable that aorta M61 exhibits higher variation of the circumferential stretch in pressure cycle $P \in[10 \mathrm{kPa} ; 16 \mathrm{kPa}]$ for higher axial prestretch $\lambda_{z Z, u L^{i n i}}$ than for smaller $\lambda_{z Z, L L} L^{i n i}$. 
Horny L, Netusil M, Vonavkova T (2014) Axial prestretch and circumferential distensibility in biomechanics of abdominal aorta. Biomechanics and Modeling in Mechanobiology 13(4):783-799. DOI: 10.1007/s10237-013-0534-8 MANUSCRIPT http://dx.doi.org/10.1007/s10237-013-0534-8

Table 2. Initial axial prestretches for donors involved in the simulation estimated with regression model (3) and its prediction intervals (4). The table is ordered in the same way as Table 1. Estimated parameters in (3) $a=2.4016$ [1/years]; $b=-0.1957$ []; and in (4) $t_{0.95 / 2}(363) \cdot S_{e}=0.0707 ; \operatorname{Mean}\left(\ln x_{i}\right)=3.8414 ;$ and $\Sigma\left(\ln x_{i}-\operatorname{Mean}\left(\ln x_{i}\right)\right)^{2}=0.0184$.

\begin{tabular}{|c|c|c|c|c|c|c|c|c|c|c|c|c|c|c|c|c|c|}
\hline ID + & F49 & F50 & F63 & F65 & M38 & M42 & M57 & M60 & M61a & M61b & M66 & M67a & M67b & M70a & M70b & M71 & M77 \\
\hline $\begin{array}{c}\lambda_{z Z^{i n i}} \\
{[-]}\end{array}$ & 1.121 & 1.117 & 1.067 & 1.061 & 1.179 & 1.156 & 1.089 & 1.078 & 1.074 & 1.074 & 1.055 & 1.055 & 1.055 & 1.046 & 1.046 & 1.043 & 1.026 \\
\hline $\begin{array}{c}\lambda_{z Z, u L^{i n i}} \\
{[-]}\end{array}$ & 1.204 & 1.199 & 1.146 & 1.139 & 1.265 & 1.240 & 1.168 & 1.157 & 1.153 & 1.153 & 1.132 & 1.132 & 1.132 & 1.123 & 1.123 & 1.119 & 1.102 \\
\hline $\begin{array}{c}\lambda_{z Z, L L^{i n i}} \\
{[-]}\end{array}$ & 1.045 & 1.041 & $0.995^{*}$ & $0.988^{*}$ & 1.098 & 1.077 & 1.014 & 1.004 & 1 & 1 & $0.983^{*}$ & $0.983^{*}$ & $0.983^{*}$ & $0.974^{*}$ & $0.974^{*}$ & $0.971^{*}$ & $0.956^{*}$ \\
\hline $\begin{array}{c}\lambda_{Z Z}\left(P_{S Y S}\right) \# \\
{[-]}\end{array}$ & 1.141 & 1.158 & 1.107 & 1.159 & 1.149 & 1.358 & 1.170 & 1.088 & 1.067 & 1.067 & 1.079 & 1.060 & 1.063 & 1.154 & 1.037 & 1.082 & 1.040 \\
\hline $\begin{array}{c}\lambda_{z Z}\left(P_{D I A}\right) \# \\
{[-]}\end{array}$ & 1.127 & 1.146 & 1.087 & 1.142 & 1.145 & 1.307 & 1.154 & 1.072 & 1.066 & 1.057 & 1.067 & 1.055 & 1.059 & 1.129 & 1.032 & 1.068 & 1.035 \\
\hline $\begin{array}{c}P_{D I A^{\S}} \\
{[\mathrm{kPa}]}\end{array}$ & 9.7 & 9.7 & 9.8 & 9.8 & 10.1 & 10.3 & 10.4 & 10.3 & 10.3 & 10.3 & 10.1 & 10 & 10 & 9.9 & 9.9 & 9.8 & 9.5 \\
\hline $\begin{array}{l}P_{S Y S} \S \\
{[\mathrm{kPa}]}\end{array}$ & 15.1 & 15.2 & 16.8 & 17 & 15.3 & 15.4 & 16.1 & 16.2 & 16.2 & 16.2 & 16.4 & 16.5 & 16.5 & 16.6 & 16.6 & 16.6 & 16.8 \\
\hline
\end{tabular}

$\dagger I D$ indicates sex (female/male) and age [years] of donors. $* \lambda_{z Z, L L}$ ini $<1$ suggests that arteries may be axially precompressed instead of prestretched. However, Horny et al. (2013b) did not report precompressed arteries in their data sample. Considering also that this values $\left(\lambda_{z Z, L L}\right.$ ini $\left.<1\right)$ occur due to the statistical methodology ( \pm deviation from expected value), $\lambda_{z Z, L L} L^{i n i}=1$ was prescribed rather than the initial precompression. \# $\lambda_{z Z}$ at $P_{S Y S}$ and PDIA computed for expected value of $\lambda_{z Z} z^{i n i}$ in the thin-walled model. §Gender specific mean values for Canadian population based on Wilkins et al. (2010).

\subsection{Circumferential and longitudinal stretch variation}

Figure 4 demonstrates how stretch variation $\mathcal{E}_{I I}(i I=\theta \Theta$ and $z Z)$ is defined. Vertical lines in the figure intersect the horizontal axis at end-points of the segments corresponding to $\left.\mathcal{E}_{i l}=\lambda_{i I}\left(P_{S Y S}\right)-\lambda_{i I}\left(P_{D I A}\right)\right)$. The stretch variations $\mathcal{E}_{i l}$ predicted by the thick-walled model (residual strains incorporated) at $r_{i}$ using expected value of the prestretch $\lambda_{z} z^{i n i}$ are depicted in Figure 5 (blue solid circles in the left upper panel). A marked decrease in $\varepsilon_{\theta \Theta}$ is even significantly correlated with age $(R=-0.572, p$-value $=0.02)$. Longitudinal systolic-diastolic stretch variation also decreases with age, but statistical significance was not attained (Figure 5, right upper panel). However, the most important fact is that the results of the simulation show that one should expect a non-zero difference in axial stretch between systolic and diastolic pressure. The range of $\varepsilon_{z Z}$ is approx. $0.005-0.025$ for expected values of $\lambda_{z} z^{i n i}$. This can be interpreted as $0.5 \%-2.5 \%$ of some reference length.

To evaluate how this result could be affected by uncertainty of the axial prestretch, $\lambda_{z Z, L L}{ }^{i n i}$ and

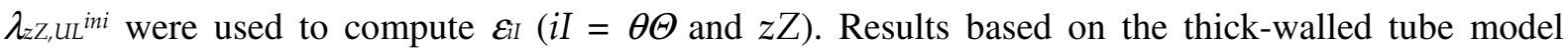
(residual strain incorporated) for both axial and circumferential direction are also presented in Figure 5. Here red open squares correspond to the upper limit (higher prestretch) and black open circles to the lower limit. Regressions lines were omitted to keep the figure clear although the results of the correlation analysis were similar to those obtained for the expected value of the prestretch. Specifically, none of the initial axial prestretch $\left(\lambda_{z Z, L L^{i n i}}\right.$ and $\left.\lambda_{z Z, U L^{i n i}}\right)$ gave significant correlation between age and $\varepsilon_{z}$, and all $\varepsilon_{\theta \Theta}$ were significantly negatively correlated with age.

Much more interesting than the numerical characterisation of the correlation with age is the position of the points. In particular, we would like to point out the difference in positions for circumferential and axial behaviour. Axial response, as can be expected, shows higher stretch variation when lower prestretch limit is used (black open circles). This is in contrast to circumferential response, 
where higher $\varepsilon_{\theta \Theta}$ is attained when the higher initial axial stretch is used (red open squares). This suggests, to the best of authors' knowledge, up to now a not yet published hypothesis that the axial prestretch not only minimises longitudinal motion of the artery upon pressure cycle, but also endows the artery with higher circumferential distensibility (in comparison with less prestretched artery characterised with the same material parameters). It is also worth noting that some of the highly prestretched aortas show diastolo-systolic shortening ( $\varepsilon_{z} z$ negative) in contrast to the weakly prestretched.

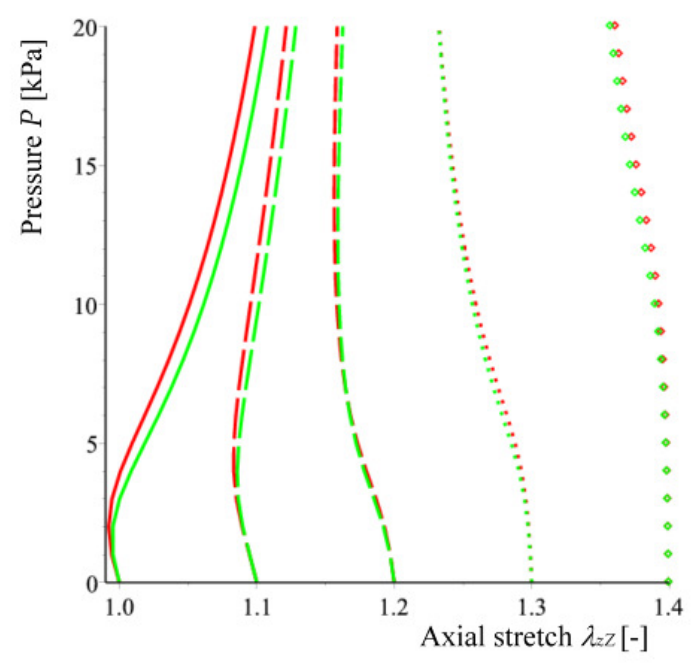

Thick-walled at $r_{i}$ Thin-walled at $r_{m}$ Thick-walled at $r_{o}$

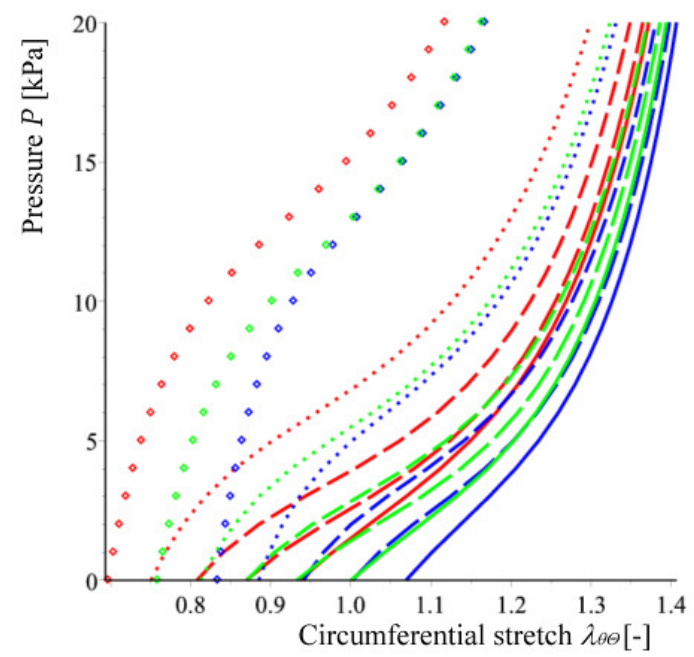

Figure 2. Inflation-extension behaviour of a 38 year old male donor (M38). The upper panel shows $P-\lambda_{z Z}$ and lower panel $P$ $\lambda_{\theta \Theta}$. Predictions for thick-walled (residual strain incorporated) model for $\lambda_{\theta \Theta}$ are computed at $r_{i}$ (red) and $r_{o}$ (blue) and results based on the thin-walled model are computed at middle radius $r_{m}$ (green). However, $\lambda z z$ is constant at all radii hence upper panel, $P-\lambda_{z} z$, includes only two colours. Each triplet $\left(P-\lambda_{\theta \Theta}\right)$ or doublet $\left(P-\lambda_{z} z\right)$ of curves corresponds to specific initial axial stretch $\lambda_{z} z^{i n i}$ $=1$ (continuous curve), 1.1 (long dashed), 1.2 (dashed), 1.3 (dotted), and 1.4 (diamonds). The easiest way to understand the panels is to consider that in $P-\lambda_{z} z$ axial prestretch increases from the left to the right, in contrast to $P-\lambda_{\theta \Theta}$ where axial prestretch increases from the right to the left. This figure manifests two basic points: (a) the axial behaviour of the tube for $P \in$ [10kPa, 16kPa] changes from axial extension (low initial axial prestretch) to axial shortening (high initial axial prestetch); and (b) the higher initial axial stretch gives $P-\lambda_{\theta \Theta}$ curves with elevated position of the inflection point (elevated on $P$-axis). Notice that while $P-\lambda_{z Z}$ curves show only small differences between computational models (thick/thin), $P-\lambda_{\theta \Theta}$ curves show that at high pressures and high axial prestretches $\lambda_{\theta \Theta}\left(r_{0}\right)$ and $\lambda_{\theta \Theta}\left(r_{m}\right)$ mutually converge more rapidly than $\lambda_{\theta \Theta}\left(r_{i}\right)$ and $\lambda_{\theta \Theta}\left(r_{m}\right)$. 
Horny L, Netusil M, Vonavkova T (2014) Axial prestretch and circumferential distensibility in biomechanics of abdominal aorta. Biomechanics and Modeling in Mechanobiology 13(4):783-799. DOI: 10.1007/s10237-013-0534-8 MANUSCRIPT http://dx.doi.org/10.1007/s10237-013-0534-8

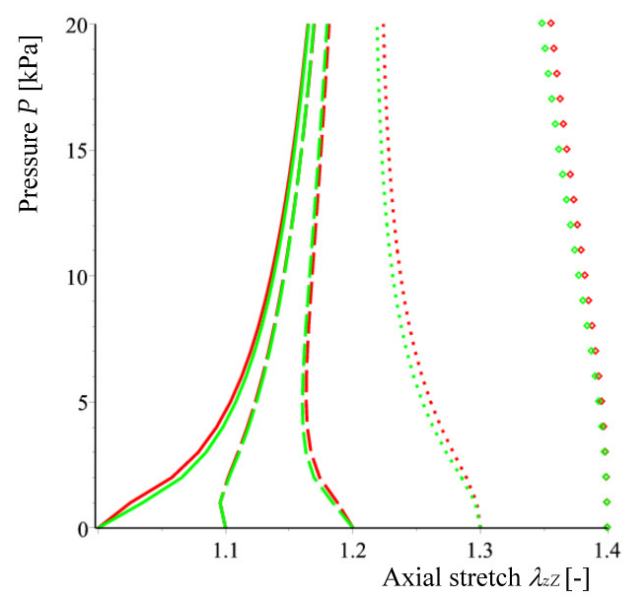

Thick-walled at $r_{i}$ Thin-walled at $r_{m}$ Thick-walled at $r_{o}$
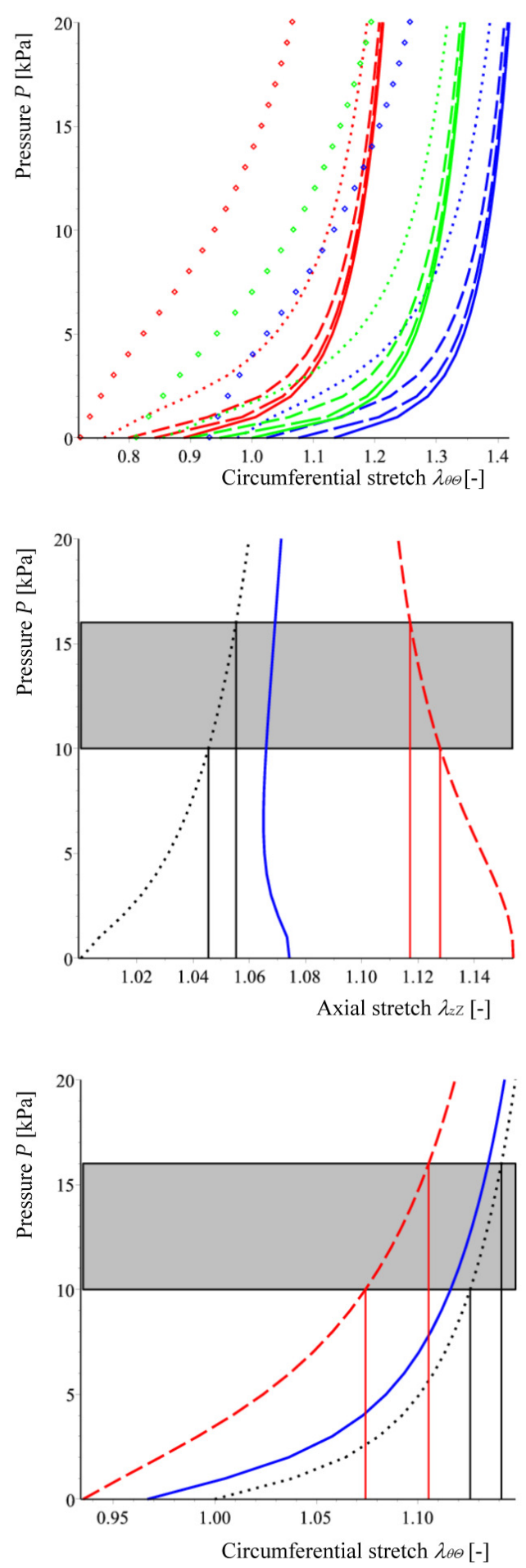

Figure 3. Inflation-extension behaviour of a 65 year old female donor (F65). The panels are arranged in the same way as in Figure 2. The graphs show two differences when compared with M38 in Figure 2. First, $P-\lambda_{z} z$ curve for $\lambda_{z} z^{i n i}=1$ does not exhibit initial shortening. It begins with axial extension. Second, the inflection point does not appear on $P-\lambda_{\theta \Theta}$ curve for $\lambda_{z} z^{i n i}=1$. However, curves for higher axial prestetch do show the inflection. Note that the existence of an inflection point makes $P-\lambda_{\theta \Theta}$ curve S-shaped and results in higher circumferential distensibility $\varepsilon_{\theta \Theta}=\lambda_{\theta \Theta}(16 \mathrm{kPa})-\lambda_{\theta \Theta}(10 \mathrm{kPa})$ (in contrast to J-shaped curve without an inflection).

Figure 4. Inflation-extension response of a 61 year old male donor (M61a) - detail. The results of the simulation based on thin-walled approximation. The blue solid curve was computed with expected value $\lambda_{z Z^{i n i}}=1.074$; red dashed curve was computed with $\lambda z Z, u L^{i n i}=$ 1.153; and black dotted curve was computed with $\lambda_{z Z, L L} L^{i n i}=1$. Shaded rectangle emphasises the region of physiological pressures. Vertical lines aid to identify stretch variation $\mathcal{E}_{i I}=\lambda_{i I}(16 \mathrm{kPa})-\lambda_{i I}(10 \mathrm{kPa})(i I=\theta \Theta$ and $z Z)$. 
Horny L, Netusil M, Vonavkova T (2014) Axial prestretch and circumferential distensibility in biomechanics of abdominal aorta. Biomechanics and

\section{$3.4 F_{\text {red }}$}

The computed reduced axial force $F_{\text {red, }}$, which generates the initial axial prestretch, is depicted in Figure 6. It is compared with values obtained for the human abdominal aorta in ex vivo elongation tests (at $P=0$ ) in Horny et al. (2013b). The figure shows the results of the simulations with expected values as well as lower and upper limit of $\lambda_{z} z^{\text {ini }}$ computed with the thin-walled model. Expected values of the initial axial stretch demonstrates that prestretching force is significantly correlated with age $(R=-0.514, p-v a l u e=0.04)$. Note that the lower limit of $\lambda_{z} z^{i n i}$ was prescribed to be 1 for donors older than 61 . In such a case $F_{\text {red }}=0$.
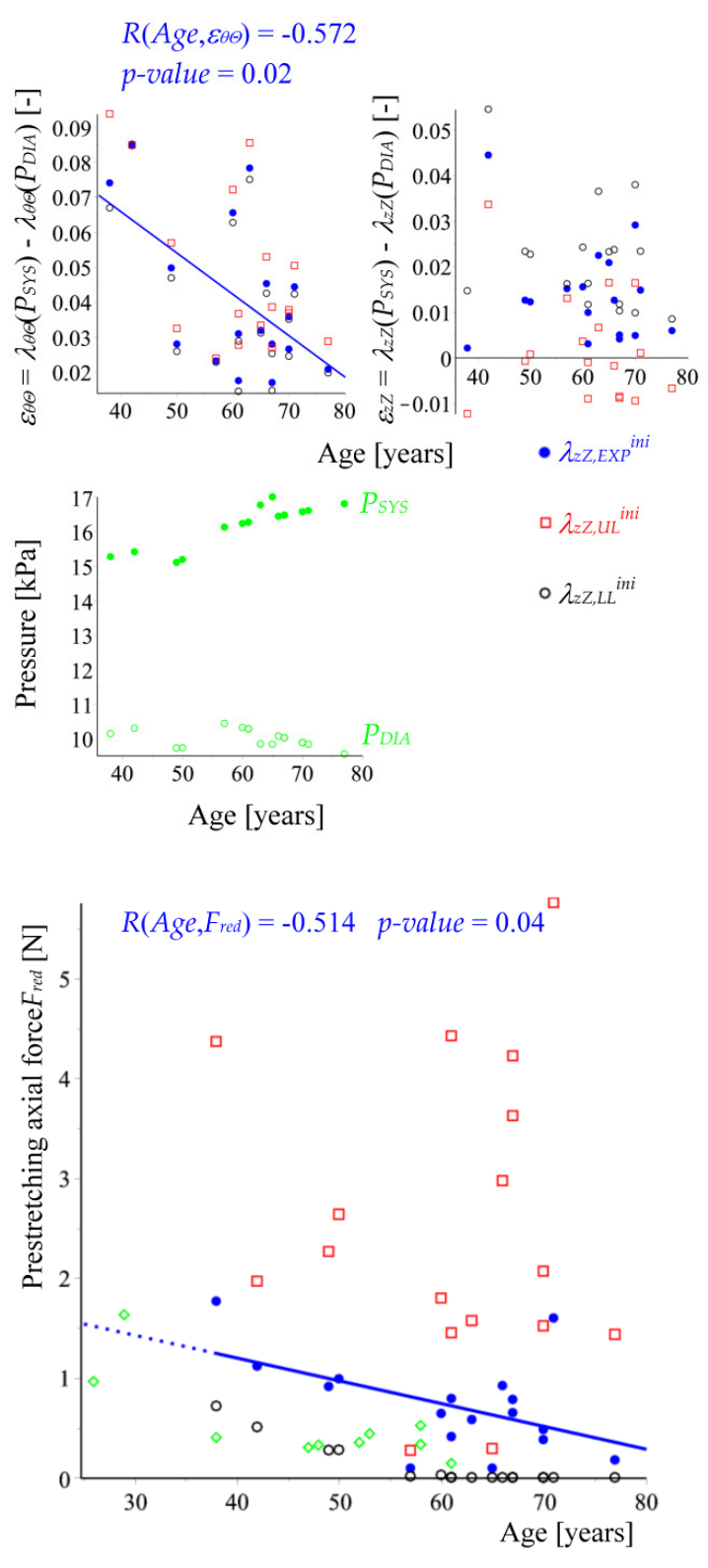

- $\lambda_{z Z, E X P}{ }^{i n i} \square \lambda_{z Z, U L}{ }^{i n i} \circ \lambda_{z Z, L L}{ }^{i n i}$

$\diamond F_{\text {red }}$ found in experiment (Horny et al., 2013b)
Figure 5. Diastolic-systolic stretch variations. The upper panels show variation of circumferential and axial stretch at $r_{i}$ and lower panel shows specific values of diastolic (PDIA) and systolic (PSYS pressure applied in the computations. Due to nonlinear large strain stiffening, $\varepsilon_{z z}$ decreases with increased axial prestretch, which is in contrast to circumferential behaviour $\left(\varepsilon_{\theta \Theta}\right.$ attained higher values for highly axially prestretched aortas).

Figure 6. Prestretching axial force. Predictions of $F_{\text {red }}$ computed with the thin-walled model are depicted: blue solid circles were obtained with expected values of $\lambda_{z} z^{i n i}$; red open squares were obtained with the upper limit; and black open circles were obtained when the lower limit of the initial axial stretch was used. The blue solid line is the linear regression model of the dependence of $F_{\text {red }}$ (obtained for expected values of the prestretch) on age: $F_{\text {red }}=2.115-0.023 \cdot$ Age for Age $\in[38 ; 77]$ years. Outside of this domain, one should consider the regression model as an extrapolation which is indicated by the dotted line. The results are compared with experiments adopted from Horny et al. (2013b). Note that due to the assumption Fred computed with $\lambda_{z Z, L L} L^{\text {ini }}$ for age $>61$ years is 0 . The regression model (4) predicts $\lambda z Z, L L^{i n i}<1$ for age $>61$ years; however, this is a consequence of the used methodology expectation \pm uncertainty. Since very little is known about initially pre-compressed arteries $\lambda_{z Z, L L}{ }^{i n i}($ Age $>61$ years $)=1$ was prescribed in our simulation. 


\subsection{Axial stress}

Almost constant axial stress during diastolic-systolic pressure variation has at times been mentioned in the literature. The predictions of (9c) for $\sigma_{z z}$ were used to evaluate this hypothesis in the present data sample. Four representative examples (M38, M57, F50, and M60) of the axial stress considering expected value (solid blue curves), upper (dashed red curves), and lower limit (black dotted curves) of the initial axial stretch are in Figure 7 drawn over circumferential stretch corresponding to $P=0 . .20$ kPa (cf. with Fig. 2 in Dobrin and Doyle 1970). Axial stress was computed employing a thick-walled model (residual strain incorporated) at the inner radius $r_{i}$. Systole and diastole are highlighted with green solid circles on the curves. It is obvious that although axial stress indeed increases slowly from the left to the right (as described in Dobrin and Doyle 1970), the diastole and systole are found on the steep part of the curves (diastolic point is always the left-hand one). This is demonstrated with M38, F57, and M60. However, F50 shows that it may not be always true.
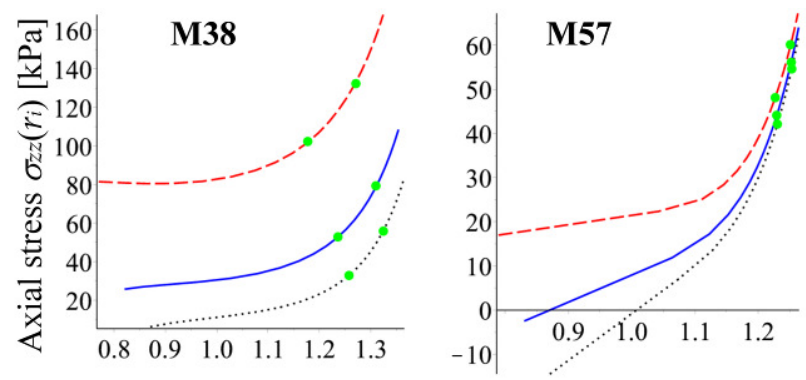

Circumferential stretch $\lambda_{\theta \Theta}\left(r_{i}\right)[-]$

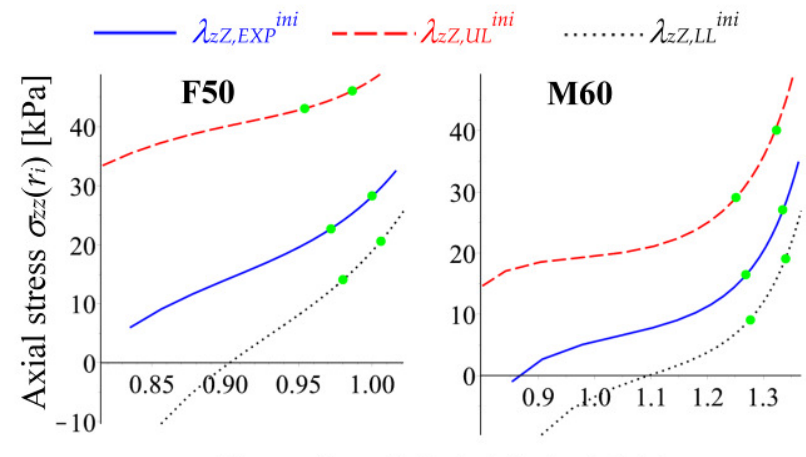

Circumferential stretch $\lambda_{\theta \Theta}\left(r_{i}\right)[-]$
Figure 7. Variation of axial stress $\sigma_{z z}\left(r_{i}\right)$ in the course of the pressurisation. The curves are based on the thickwalled model with incorporated residual strain. Circumferential stretch on horizontal axis starts from values smaller than 1 due to simultaneous effect of the initial axial stretch and residual strain. Predictions obtained with the expected value of $\lambda_{z} z^{i n i}$ are depicted with blue solid curves; red dashed curves were obtained with $\lambda_{z Z, U L}{ }^{i n i}$; and $\lambda_{z Z, L L}{ }^{i n i}$ was used to create black dotted curves. Solid circles on the curves highlight the positions of diastolic (the left circle) and systolic (right circle) pressure.

To quantify diastolic-systolic increment in $\sigma_{z z}$ numerically, Figure 8 depicts the stress increment normalised with respect to the diastole for all involved donors. The upper panel shows increments obtained with the thick-walled model with incorporated residual strain for $\sigma_{z z}\left(r_{i}\right)$ and $\sigma_{z z}\left(r_{o}\right)$, and lower panel was obtained in the thin-walled approximation (mean axial stress at $r_{m}$ ). The symbols are used in the same way as in the previous figures (to distinguish inner and outer radius, small and large symbols are used, respectively). It is clear from the figure that the lowest changes of the axial stress are obtained using the upper limit of the initial axial stretch. The same is confirmed in the thin-walled model. The lower panel shows that average change of $\sigma_{z z}$ is in the range $0.2-0.5$ for highly prestretched aortas whereas weakly prestretched aortas show average change of approx. 0.7. 
Significant correlation between variation of axial stress and age was obtained at $r_{m}$ and $r_{o}$ and the lower the initial prestretch, the higher correlation coefficient was obtained (the highest one, $R=0.790 p$-value $<0.001$, was at $r_{o}$ ).

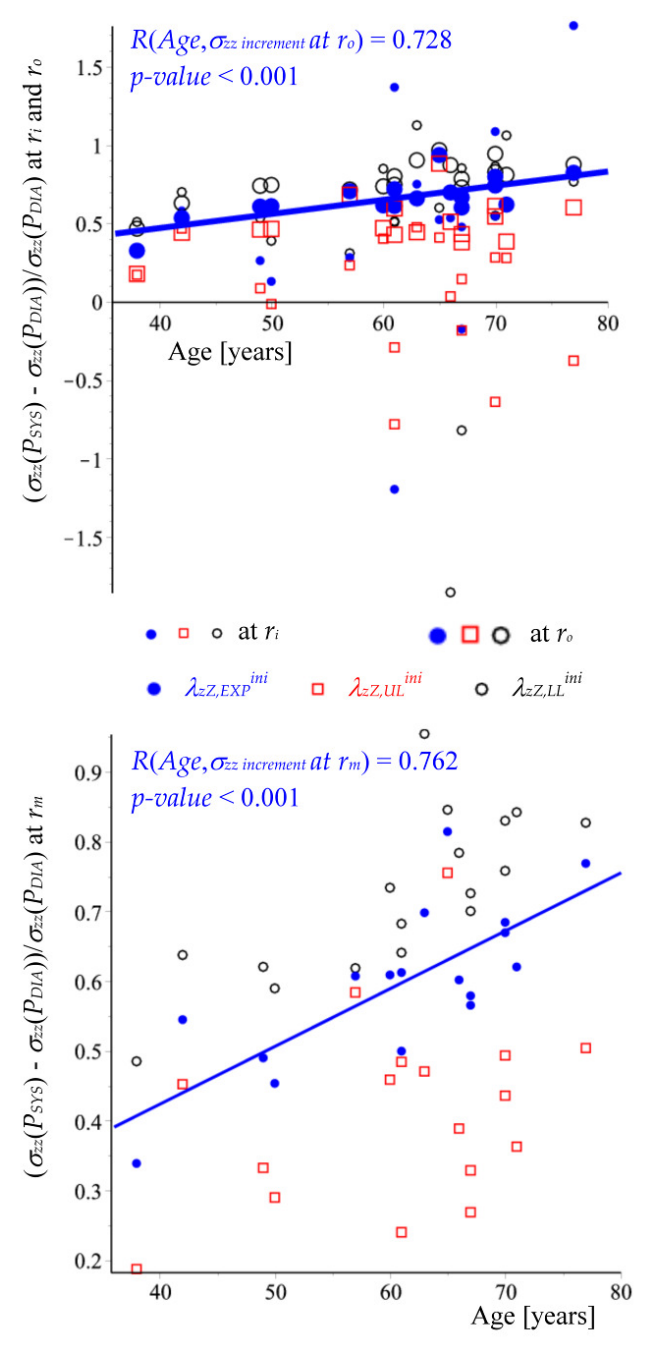

Figure 8. Relative change of the axial stress induced by diastolicsystolic pressure increment. The upper panel shows the results computed with the thick-walled model (residual strain incorporated) at the inner radius (small symbols) and at the outer radius (large symbols). The lower panel shows results obtained by thin-walled approximation. The symbols are used in the same way as in Figure 5 and 6. The figure shows that higher initial axial prestretch is accompanied with smaller changes of the axial stress. Correlation coefficients and regression lines correspond to expected initial prestretch.

\subsection{Stiffness ratio $C_{\Theta \Theta \Theta \Theta} / C_{Z Z Z Z}$}

The ratio between components of elasticity tensor was computed for all involved individuals. The results are summarised in Figures 9, 10 and 11. To show an order of the magnitude of the components of elasticity tensor, numeric values are compared in Figure 9 for inner, outer, and middle radius of the aortas initially prestrained to the expected axial prestretch and pressurised to PDIA and PSYS. Significant correlation between age and the components of $\mathbf{C}$ were only found in case of $C_{\Theta \Theta \Theta \Theta}$ computed at $r_{m}$ for $P_{S Y S}$ at all prestretches $(R \approx 0.52 p$-value $\approx 0.04)$. All other cases were not significant.

The stiffness ratio $C_{\Theta \Theta \Theta \Theta} / C_{Z z Z z}$ is depicted in Figure 10. All effects are herein summarised - the effect of finite thickness of the wall; effect of the pressure; and the effect of initial prestretch. Two facts are demonstrated by Figure 10. First, the stiffness ratio depends strongly on radial position within the thickness of the artery wall. In average $C_{\Theta \Theta \Theta \Theta} / C_{\mathrm{zzzZ}}<1$ is at the inner radius (top panels in Figure 
10 ), however $C_{\Theta \Theta \Theta \Theta} / C_{z z z z}>1$ holds at outer radius (bottom panels). Second, with regard to the effect of the prestretch, thin-walled model for $r_{m}$ and thick-walled model at $r_{o}$ show that higher prestretch is accompanied with the lower stiffness ratio (and reciprocally lower prestretch with the higher stiffness ratio). However, there are some cases at the inner radius which deviate from this rule. Correlation analysis revealed significant dependence of the stiffness ratio on age only in case of thin-walled model. It was found $R=0.514$ with $p$-value $=0.04$ for $P_{S Y S}$ and $P_{D I A}$ at expected initial axial stretch.

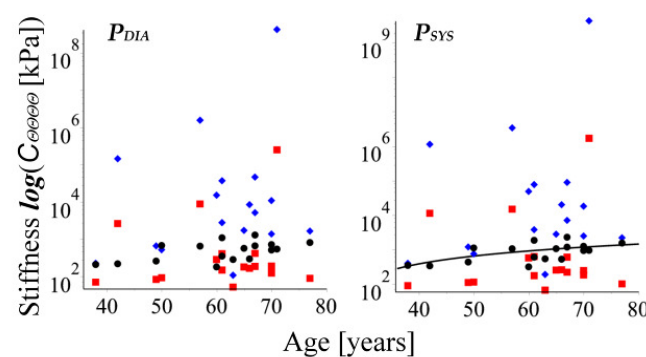

- THICK-WALLED MODEL INNER RADIUS

- THIN-WALLED MODEL MIDDLE RADIUS

- THICK-WALLED MODEL OUTER RADIUS

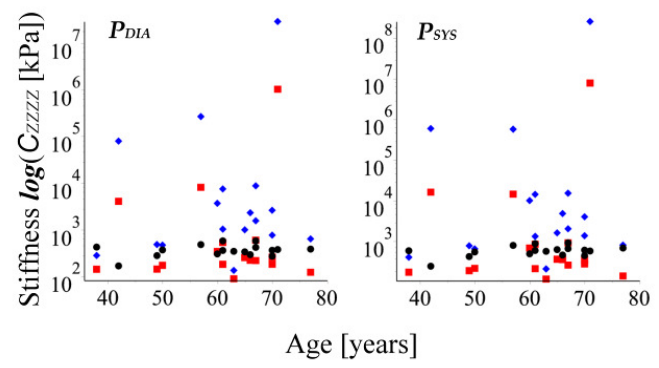

Figure 9. Components of referential elasticity tensor $\mathbf{C}$ for expected axial prestretch. Upper panels show the stiffness in circumferential direction $\left(C_{\Theta \Theta \Theta \Theta}\right)$ and lower panels in axial direction $(\mathrm{Czzzz})$. The symbols indicate the method and position: red solid boxes - at $r_{i}$ with thick-walled model; black solid circles at $r_{m}$ with thin-walled model; and blue solid diamonds - at $r_{o}$ with thick-walled model. The regression line indicates significant correlation between age and $C_{\Theta \Theta \Theta \Theta}$ at $r_{m}$ for $P_{S Y S}(R=0.515 p$ value $=0.04)$. Note that logarithmic scale is used on vertical axes.

Relative increments of the stiffness ratio induced by diastolic-systolic variation of internal pressure are depicted in Figure 11. The increments are normalised with respect to the ratio at diastolic pressure. In contrast to the stiffness ratio as such, highly prestretched aortas showed higher relative increments of the stiffness ratio from diastole to systole than weakly prestretch aortas. Significant negative correlation with age was found in the case of relative increments computed with the thickwalled model at inner radius $(R=-0.600 p$-value $=0.02$ for expected prestretch, and $R=-0.539 p-$ value $=0.03$ for upper axial prestretch). 

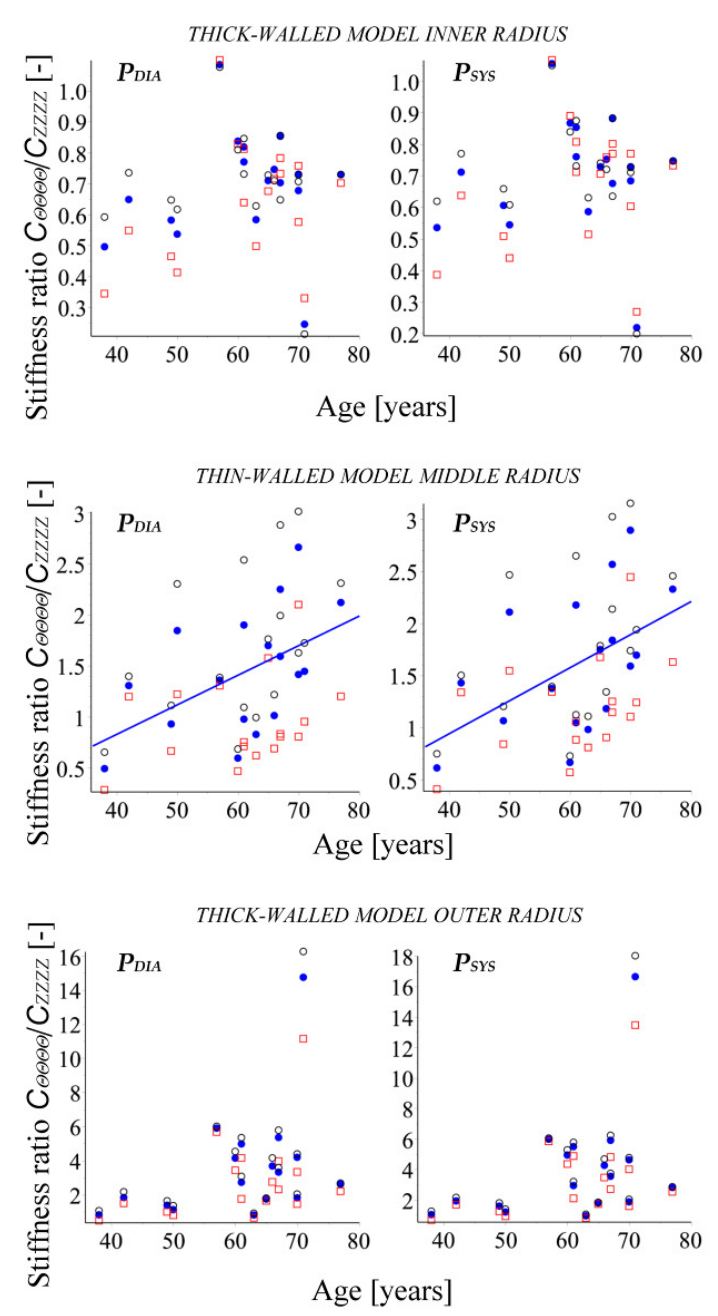

- $\lambda z Z, \operatorname{EXP}^{\text {ini }} \quad \square \lambda_{z Z, U L}{ }^{\text {ini }} \quad \circ \lambda_{z Z, L L}{ }^{i n i}$

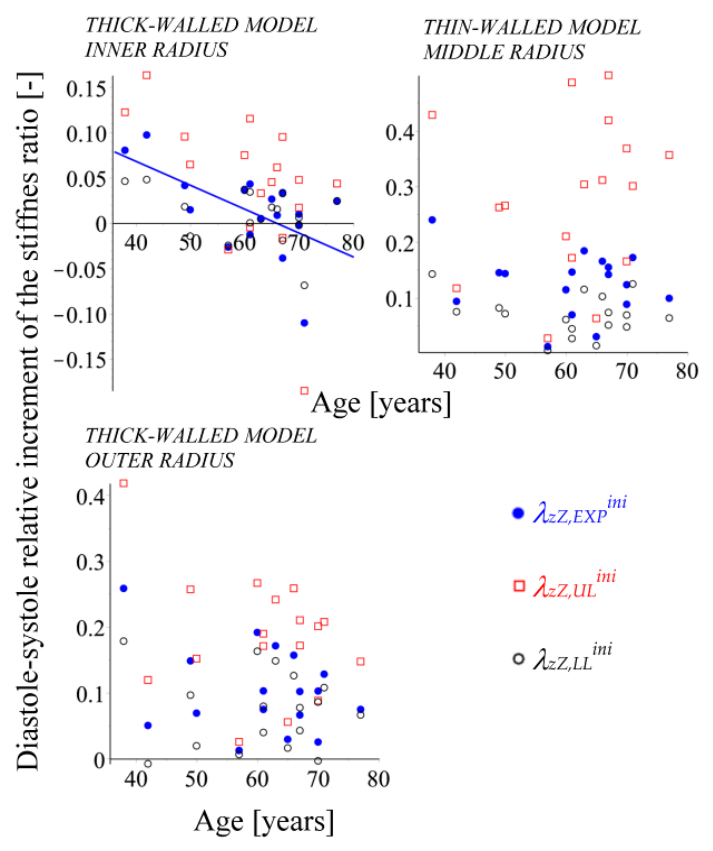

Figure 10 Stiffness ratio. The figure summarises results obtained for stiffness ratio at inner, middle and outer radius of the aortas. Three important things can be derived from the figure: (1) weakly prestretched aortas give higher stiffness ration; (2) the stiffness ratio varies significantly through the thickness of the wall; and (3) aortas may exhibit different stiffness ratios in different ageing periods.

Figure 11. Relative increment of the stiffness ratio. Highly prestretched aortas gave a higher relative increment in the stiffness ratio during pressure cycle. 
Horny L, Netusil M, Vonavkova T (2014) Axial prestretch and circumferential distensibility in biomechanics of abdominal aorta. Biomechanics and Modeling in Mechanobiology 13(4):783-799. DOI: 10.1007/s10237-013-0534-8 MANUSCRIPT http://dx.doi.org/10.1007/s10237-013-0534-8

\section{Discussion}

Our study focused on the effect of age-related decrease in the initial longitudinal prestretch of human abdominal aorta on its distensibility, axial stress, and circumferential-to-axial stiffness ratio. Changes induced by the variation of the pressure (systole - diastole) were also evaluated. Since our bibliographic search did not find a complete description of human abdominal aorta anywhere (i.e. specimens with documented geometry, experimentally determined constitutive parameters and axial prestretch in one study), the characteristics of arteries were adopted from two different papers. Geometry and constitutive description were taken from Labrosse et al. (2013) who conducted experimental ex vivo inflation (with free axial extension) of the human abdominal aorta. Statistics of the axial prestretch in the same anatomical location were reported by Horny et al. (2013a) who performed autopsy measurement on the sample of 365 human cadavers. Since this approach induces some uncertainty in the true value of the prestretch, all computations were performed with expected prestretch (i.e. prestretch exactly corresponding to the regression equation (3)), and also with upper and lower limit of 95\%-confidence interval of a prediction (so-called prediction interval; equation (4)).

Our study modelled the inflation-extension response by considering the aorta to be a prestrained, anisotropic and nonlinear homogenous tube with closed ends by the methods of elastostatics. Two analytical approaches were used. First was the thin-walled model which operates with mean wall stresses acting at middle radius of the tube and its results were considered to be basic estimates of the mechanical response. However, this model cannot capture the effect of residual strain on the stress state of an artery. To this end, the thick-walled model with incorporated residual strain was also used in the situations when transmural distribution of quantities was of interest.

\subsection{Stretch variation of prestretched artery}

The results suggest that, although axial prestretch significantly decreases due to ageing (Figure 1), it can crucially affect mechanical response. This fact is clearly observable in systolic-diastolic variation of the circumferential stretch $\varepsilon_{\theta \Theta}$. Circumferential stretch variation was found to decline with age (Figure 5), but our study demonstrated that highly prestretched arteries $\left(\lambda_{z z, u L^{i n i}}\right)$ can be more distended in the circumferential direction by the same internal pressure in comparison with their less prestretched $\left(\lambda_{z Z, L L}{ }^{i n i}\right)$ counterparts. This property was revealed in the thick-walled (at $r_{i}$ and $\left.r_{o}\right)$ and also in the thin-walled (at $r_{m}$ ) model. The results were qualitatively similar, hence, the variation only at the inner radius is presented (Figure 5).

To the best of authors' knowledge, preceding studies have not pointed out this fact which might certainly warrant re-examination in the future (experiments are necessary to validate this finding). Results suggest that axial prestretch could play a more important role than merely a way how to endow abdominal aorta with a property of almost constant axial length during the pressure cycle (Dobrin 1978; Dobrin et al. 1990; van Loon et al. 1977). We should note there are studies reporting results from which a similar conclusion could be obtained, although they are focused on different anatomical locations. However, their authors did not investigate this property in detail; cf. Figure 5 in Sommer et al. (2010) and Figure 4 in Sommer and Holzapfel (2012) for human carotid artery, and also Figure 4 in Schulze-Bauer et al. (2003) for human iliac artery. 
Horny L, Netusil M, Vonavkova T (2014) Axial prestretch and circumferential distensibility in biomechanics of abdominal aorta. Biomechanics and Modeling in Mechanobiology 13(4):783-799. DOI: 10.1007/s10237-013-0534-8 MANUSCRIPT http://dx.doi.org/10.1007/s10237-013-0534-8

Possible explanation of this interesting fact is that the axial prestretch may align collagen fibres (main load-bearing component of artery wall responsible for arterial anisotropy; Holzapfel et al. 2000) to axial direction and the wall subsequently shows higher stretch variation in the circumferential direction. This hypothesis is in accordance with the stiffness ratio in Figure 10. Here open black circles (weak prestretch prescribed to aortas) most frequently lie above red open squares (high prestretched prescribed). That is to say that the increase in the prestretch leads to the decrease of the circumferential-to-axial stiffness ratio.

Considering "constancy" of the length, Figures 5 suggests that this is "only" an approximation. The simulation indicates axial stretch variation may be expected in the range $\mathcal{E}_{z z}=-0.01-0.04$, depending on the specific initial axial prestretch. The right panel in Figure 5 shows we should expect higher change in axial stretch during pressure cycle when lower initial axial prestretch is applied (black open circles are the most distant from horizontal axis). On the contrary, the upper limit of initial axial stretch led to $\varepsilon_{z z}$ located closely to the horizontal axis. In some cases, the model predicts a negative value which is the shortening of the artery when pressurised from diastole to systole. However, these results come from simulation, not experiment.

We should avoid over-interpretation of negative/positive $\varepsilon_{z} z$ in specific cases; nevertheless, higher initial prestrech led to $\varepsilon_{z} z=-0.01-0.02$, in contrast to lower prestretch which gave $\varepsilon_{z z}=0.01-0.04$. Thus, using higher $\lambda_{z} z^{i n i}$ is in accordance with the property of the relatively constant length of the aorta mentioned in the literature (Dobrin 1978; Dobrin et al. 1990; van Loon et al. 1977). Nevertheless, in the case that age-related changes leading to the loss of the prestretch progress rapidly (i.e. specific $\lambda_{z} z^{i n i}$ is close to the lower limit of the prediction interval), the simulation suggests we should expect that the property of almost constant length may be lost. Moreover, considering $\varepsilon_{z z}$ as engineering strain, changes of about $2-4 \%$ could fall into the range measurable by modern imaging (e.g. ultrasound) methods (Ahlgren et al. 2012; Cinthio et al. 2006; Karatolis et al. 2013; Larsson et al. 2011).Therefore, since the lower prestretch corresponds to higher axial distension in pressure cycle (intra-pressure cycle deformation), it seems to open a new diagnostic possibility based on longitudinal strain measurement governed by the hypothesis that high (for instance higher than $2 \%$ ) intra-cycle axial stretch variation may suggest suboptimal axial prestretch. The word "optimal" is used not only with respect to the implication "minimal change in axial stretch during pressure cycle gives higher circumferential distensibility, which supports a windkessel function", but also with respect to the hypothesis that no change in axial length implies no energetic demand for axial displacements (Schulze-Bauer et al. 2003) and consequently no dissipation of this energy due to viscoelasticity of the artery wall.

\subsection{Axial stress and prestretching force}

The simulation confirms our previous conclusion (Horny et al. 2013b) that prestretching axial force (force induced by $\lambda_{z} z^{i n i}$ ) decreases with age (statistical significance attained). Specific values are slightly higher than in Horny et al. (2013b), see Figure 7. The upper limit of the initial axial prestretch in some cases did indeed lead to high force $\left(F_{\text {red }}>3 \mathrm{~N}\right)$.

The simulation also confirmed that the $\sigma_{z z}-\lambda_{\theta \Theta}$ relationship is initiated with very slow stress increment; c.f. Figure 7 with Figure 2 in Dobrin and Doyle (1970). However, the positions of diastolic 
and systolic pressure (indicated with green solid circles on the curves in Figure 7) do not correspond with Dobrin and Doyle's conclusion that axial stress should be almost constant during the pressure cycle. It is more clearly evident in Figure 8 where normalised diastolic-systolic increments of axial stress are depicted. Depending on the specific prestretch, the simulation suggests we should expect a variation of mean axial stress (lower panel) in the order of tens of percentage. The fundamental role of sufficient axial prestretch is again clear. The substitution of upper-limit values into the calculation led to the smallest increments. This suggests that insufficiently prestretched arteries, because they feel a larger change in axial stress during pressure cycle, may not operate in physiologically optimal conditions and could be vulnerable to a mechano-biological reaction attempting to restore homeostasis because changes in the axial stress/strain state have been identified as quantities initiating a remodelling (Humphrey et al. 2009; Jackson et al. 2002; Lawrence and Gooch 2009). The discrepancy with the observation made by Dobrin and Doyle (1970) may be attributed to the fact that they conducted their experiments with relatively young and healthy laboratory dogs.

An age-related decrease in the prestretch leads to the decrease in the prestretching force and consecutively it leads to decreased initial axial stress. This can be concluded from Figure 7 when $\sigma_{z z}\left(r_{i}\right)$ corresponding to $P=0$ (starting points of the curves) is considered. When axial prestretch is applied, curves do not begin at $\lambda_{\theta \Theta}=1$ (decrease in the radius accompanies initial axial extension). Interestingly, some of curves initiate with negative values of axial stress at $r$. This is the effect of the residual strain which, for high opening angles, qualitatively change transmural stress distribution (see Figure 7 in Labrosse et al. 2013). In fact, when residual stress is released in a radial cut of an artery and the arterial ring springs to the open sector, a small axial deformation occurs. The prestretch induced by $F_{\text {red }}$ is superimposed on this small axial deformation. Figure 7 thus documents that the small values of $F_{\text {red, }}$, which accompanies small $\lambda_{z} z^{i n i}$, with a simultaneous occurrence of high opening angle (see Table 1 for specific values) can result in negative axial stress at $r_{i}$ in non-pressurised but axially prestretched artery (for the effect of residual strain on closed, non-prestretched and nonpressurised artery see e.g. Figure 2 in the review Rachev and Greenwald 2003; or in Chuong and Fung 1986). This fact, to the best of our knowledge, has also not been previously mentioned in the literature. Nevertheless, we should point out that this configuration (axial prestretch superimposed on residually stressed artery with no luminal pressure) is never attained in vivo.

\subsection{Stiffness ratio}

Figures 9, 10 and 11 depict results obtained for components of the elasticity tensor, their circumferential-to-axial ratio, and relative diastolo-systolic increment in the stiffness. In contrast to axial stress, the ratio seems to satisfy more closely the condition of constancy during pressurisation (Figure 11). This suggests that the stiffness ratio could be more suitable for purposes of in vivo parameters estimation where some assumption has to be made to overcome the impossibility to directly measure axial force and stress (Chen et al. 2008). Figure 10 (middle panels), however, shows that this ratio may not be constant during ageing. Moreover, the results of thick-walled model show that $C_{\Theta \Theta \Theta \Theta} / C_{z z z z}$ depends on radial position within the thickness of artery wall (e.g. the stiffness ratio at $P_{S Y S}$ and expected $\lambda_{z Z} z^{i n i}$ was found to be $0.701 \pm 0.182$ at $r_{i}, 1.60 \pm 0.657$ at $r_{m}$, and $3.96 \pm 3.70$ at $r_{o}$; mean $\pm \mathrm{SD}$ ). The results of thick-walled model (residual strain incorporated) suggest that the aortic 
Horny L, Netusil M, Vonavkova T (2014) Axial prestretch and circumferential distensibility in biomechanics of abdominal aorta. Biomechanics and Modeling in Mechanobiology 13(4):783-799. DOI: 10.1007/s10237-013-0534-8 MANUSCRIPT http://dx.doi.org/10.1007/s10237-013-0534-8

wall in cardiac cycle is stiffer in axial direction at its inner radius; however, at outer radius it is stiffer in circumferential direction. This is the consequence of non-homogenous strain distribution over the thickness of the artery.

\subsection{Axial prestretch}

Axial prestretch as such and its age-related changes was not the main subject of our study because it has previously been presented elsewhere (Horny et al. 2011, 2012a,b, 2013a). Nevertheless, we would like to explicitly emphasise three things. First, our study used the same regression model of $\lambda_{z} z^{\text {ini-age }}$ in the case of both genders because it was previously found that significant differences in the prestretch of abdominal aorta between males and females do not exist (Horny et al. 2012b). In that same paper, the authors proved assumptions of the classical linear regression model which is important for construction of the prediction intervals. Finally, according to Horny et al. (2013a), we do not expect that the post mortem interval and atherosclerotic changes in abdominal aorta can significantly deviate used estimates of $\lambda_{z} z^{\text {ini }}$ from their true values (in fact unknown) at time of the death.

\subsection{Effect blood pressure uncertainty}

In the simulation, mean (but age and gender specific) diastolic and systolic pressures adopted from Wilkins et al. (2010) were applied. Used pressures however are only estimates of the true pressures sustained by donors in their life. To eliminate the possible effect of varying quality of medical care in different countries, blood pressures were adopted from very recent survey conducted in the same country (Canada) as the tissue donors came from. For the sake of clarity, we decided to do not complicate it with another quantity considered with uncertainty (blood pressure). This is motivated by two following facts. First, in future it would be better to verify our results in experiments and our article should function as initial motivation. Second, one can, although only roughly, estimate how the results will change with changed $P_{D I A}$ and $P_{S Y S}$. Consider that changes in $P_{D I A}$ and $P_{S Y S}$ can be understood as a movement in the vertical direction of the shaded rectangle in Figure 4 (one can also draw such a rectangle into Figure 2 and 3). The figure is created for the range of $0-20 \mathrm{kPa}$. It is clear that the positive effect of the prestretch on the stretch variation in the circumferential direction is restricted by a monotony of $d P / d \lambda_{\theta \Theta}$. This is most clearly seen in Figure 2 (lower panel) when the highest prestretch (red diamonds) is considered. When $P_{D I A}$ decreases less than approx. $5 \mathrm{kPa}$, the positive effect of the prestretch is lost due to the increasing slope of the curve (reciprocally an increase of $P_{S Y S}$ to $\approx 20 \mathrm{kPa}$ has the same effect). This suggests that to reach maxim circumferential stretch variation, it would be optimal for an artery to operate close to the inflection point on $P-\lambda_{\theta \Theta}$. This position, however, depends on specific numerical values of the constitutive parameters. 
Horny L, Netusil M, Vonavkova T (2014) Axial prestretch and circumferential distensibility in biomechanics of abdominal aorta. Biomechanics and Modeling in Mechanobiology 13(4):783-799. DOI: 10.1007/s10237-013-0534-8 MANUSCRIPT http://dx.doi.org/10.1007/s10237-013-0534-8

\subsection{Limits of the simulation}

Our study has limitations coming from (a) the chosen method, and (b) from the data used. Firstly, it should be pointed out that the elastostatics approach was used. This means that the presented simulations correspond to the so-called inflation-extension experiment (the most frequent way of the experimental constitutive model determination for cylindrical segments of arteries), but true in vivo arterial mechanics consists in pressure pulse wave propagation as a result of dynamical fluid-structure interaction. The chosen approach, however, mimics the methods used in in vivo parameters estimation procedures presented in recent literature (Åstrand et al. 2011; Masson et al. 2008, 2011; Schulze-Bauer and Holzapfel 2003; Stålhand 2009; Stålhand and Klarbring 2005, Wittek et al. 2013).

It should also be mentioned that recent papers have proven that residual strains in the artery differ with respect to its layered structure. This fact is not captured in the simulation because it is based on the assumption of a homogenous wall (adopted from Labrosse et al. 2013). Layered structure, theoretically, may induce discontinuities and non-smoothness in transmural stress and strain distribution; see e.g. Figure 19 in Holzapfel et al. (2000); Figure 5 in Holzapfel and Ogden (2010b). It might affect results obtained with a thick-model.

\section{Conclusion}

The above presented calculations have shown that, although ageing led to significantly decreased longitudinal prestretch, the biomechanical response of the human abdominal aorta was changed significantly depending on used initial axial stretch within the computation. Particularly, substituting the upper limit of the confidence of prediction for the initial axial prestretch gave mechanical responses which can be characterised by (a) lower variation in axial length, and (b) higher circumferential distensibility, in contrast to the responses obtained for arteries with low initial axial prestretch. The simulation also showed a significant effect of the axial prestretch on the variation of mean axial stress during the pressure cycle. Finally, the obtained results are in accordance with the hypothesis that the circumferential-to-axial stiffness ratio is the quantity relatively constant within this cycle.

\section{Acknowledgement}

This work has been supported by the Czech Technical University in Prague under project SGS13/176/OHK2/3T/12, Czech Ministry of Health project NT 13302, Technology Agency of the Czech Republic in the project TA 01010185, and by the Faculty of Mathematics and Physics of Charles University in Prague project no. 267310.

\section{References}

Ahlgren TR, Cinthio M, Persson HW, Lindström K (2012). Different patterns of longitudinal displacement of the common carotid artery wall in healthy humans are stable over a four-month period. Ultrasound Med Biol 38:916-925. doi: 10.1016/j.ultrasmedbio.2012.02.005

Åstrand H, Stålhand J, Karlsson J, Karlsson M, Sonesson B, Länne T (2011) In vivo estimation of the contribution of elastin and collagen to the mechanical properties in the human abdominal aorta: Effect of age and sex. J Appl Physiol 110:176187. doi: 10.1152/japplphysiol.00579.2010 
Horny L, Netusil M, Vonavkova T (2014) Axial prestretch and circumferential distensibility in biomechanics of abdominal aorta. Biomechanics and Modeling in Mechanobiology 13(4):783-799. DOI: 10.1007/s10237-013-0534-8 MANUSCRIPT http://dx.doi.org/10.1007/s10237-013-0534-8

Carta L, Wagenseil, JE, Knutsen RH, Mariko B, Faury G, Davis EC, et al. (2009) Discrete contributions of elastic fiber components to arterial development and mechanical compliance. Arterioscler Thromb Vasc Biol 29:2083-2089. doi: 10.1161/ATVBAHA.109.193227

Chen K, Fata B, Einstein DR (2008) Characterization of the highly nonlinear and anisotropic vascular tissues from experimental inflation data: A validation study toward the use of clinical data for in-vivo modeling and analysis. Ann Biomed Eng 36:1668-1680. doi: 10.1007/s10439-008-9541-9

Chuong CJ, Fung YC (1986) On residual stresses in arteries. J Biomech Eng 108:189-92.

Cinthio M, Ahlgren ÅR, Bergkvist J, Jansson T, Persson HW, Lindström K (2006). Longitudinal movements and resulting shear strain of the arterial wall. Am J Physiol Heart Circ Physiol 291:H394-H402. doi: 10.1152/ajpheart.00988.2005

Dobrin PB (1978) Mechanical properties of arteries. Physiol Rev 58:397-460

Dobrin PB, Doyle JM (1970) Vascular smooth muscle and the anisotropy of dog carotid artery. Circ Res 27:105-119

Dobrin PB, Schwarcz TH, Mrkvicka R (1990) Longitudinal retractive force in pressurized dog and human arteries. J Surg Res 48:116-120. doi: 10.1016/0022-4804(90)90202-D

Greenwald SE (2007) Ageing of the conduit arteries. J Pathol 211:157-172. doi: 10.1002/path.2101

Guccione JM, McCulloch AD, Waldman LK (1991) Passive material properties of intact ventricular myocardium determined from a cylindrical model. J Biomech Eng 113:42-55. doi: 10.1115/1.2894084

Han HC, Fung YC (1995) Longitudinal strain of canine and porcine aortas. J Biomech 28:637-641. doi: 10.1016/00219290(94)00091-H

Holzapfel GA, Gasser TC, Ogden RW (2000) A new constitutive framework for arterial wall mechanics and a comparative study of material models. J Elast 61:1-48. doi: 10.1023/A:1010835316564

Holzapfel GA (2000) Nonlinear solid mechanics: A continuum approach for engineering. Wiley \& Sons, Chichester.

Holzapfel GA, Ogden RW (2010a) Constitutive modelling of arteries. Proc R Soc A 466:1551-1597. doi: 10.1098/rspa.2010.0058

Holzapfel GA, Ogden RW (2010b) Modelling the layer-specific three-dimensional residual stresses in arteries, with an application to the human aorta. J R Soc Interface 7:787-99. doi: 10.1098/rsif.2009.0357

Horny L, Adamek T, Gultova E, Zitny R, Vesely J, Chlup H, Konvickova S (2011) Correlations between age, prestrain, diameter and atherosclerosis in the male abdominal aorta. J Mech Behav Biomed Mater 4:2128-2132. doi: 10.1016/j.jmbbm.2011.07.011

Horny L, Adamek T, Vesely J, Chlup H, Zitny R, Konvickova S (2012a) Age-related distribution of longitudinal pre-strain in abdominal aorta with emphasis on forensic application. Forensic Sci Int 214:18-22. doi: 10.1016/j.forsciint.2011.07.007

Horny L, Adamek T, Chlup H, Zitny R (2012b) Age estimation based on a combined arteriosclerotic index. Int J Leg Med 126:321-326. doi: 10.1007/s00414-011-0653-7

Horny L, Adamek T, Kulvajtova M (2013a) Analysis of axial prestretch in abdominal aorta with reference to post mortem interval and degree of atherosclerosis. J Mech Behav Biomed Mater, in press. doi: 10.1016/j.jmbbm.2013.01.033

Horny L, Adamek T, Zitny R (2013b) Age-related changes in longitudinal prestress in human abdominal aorta. Arch Appl Mech 83:875-888. doi: 10.1007/s00419-012-0723-4

Humphrey JD (2002) Cardiovascular solid mechanics: Cells, tissues and organs. Springer-Verlag, New York

Humphrey JD, Eberth JF, Dye WW, Gleason RL (2009) Fundamental role of axial stress in compensatory adaptations by arteries. J Biomech 42:1-8. doi: 10.1016/j.jbiomech.2008.11.011

Jackson ZS, Gotlieb AI, Langille BL (2002). Wall tissue remodeling regulates longitudinal tension in arteries. Circ Res 90:918-925. doi: 10.1161/01.RES.0000016481.87703.CC

Kalita P, Schaefer R (2008) Mechanical models of artery walls. Arch Comput Methods Eng 15:1-36. doi: 10.1007/s11831007-9015-5

Karatolios K, Wittek A, Nwe TH, Bihari P, Shelke A, Josef D, Schmitz-Rixen T, Geks J, Maisch B, Blase C, Moosdorf R, Vogt S. (2013) Method for aortic wall strain measurement with three-dimensional ultrasound speckle tracking and fitted finite element analysis. Ann Thorac Surg, in press. doi: 10.1016/j.athoracsur.2013.06.037

Labrosse MR, Beller CJ, Mesana T, Veinot JP (2009) Mechanical behavior of human aortas: Experiments, material constants and 3-D finite element modeling including residual stress. J Biomech 42:996-1004. doi: 10.1016/j.jbiomech.2009.02.009

Labrosse MR, Gerson ER, Veinot JP, Beller CJ (2013) Mechanical characterization of human aortas from pressurization testing and a paradigm shift for circumferential residual stress. J Mech Behav Biomed Mater 17:44-55. doi: 10.1016/j.jmbbm.2012.08.004

Langewouters GJ, Wesseling KH, Goedhard WJA (1984) The static elastic properties of 45 human thoracic and 20 abdominal aortas in vitro and the parameters of a new model. J Biomech 17:425-435.

Larsson M, Kremer F, Claus P, Kuznetsova T, Brodin L, D'Hooge J (2011) Ultrasound-based radial and longitudinal strain estimation of the carotid artery: A feasibility study. IEEE Trans Ultrason Ferroelectr Freq Control 58(10), 2244-2251. doi: 10.1109/TUFFC.2011.2074

Lawrence AR, Gooch KJ (2009) Transmural pressure and axial loading interactively regulate arterial remodeling ex vivo. Am J Physiol Heart Circ Physiol 297:H475-H484. doi: 10.1152/ajpheart.00972.2008

Learoyd BM, Taylor MG (1966) Alterations with age in the viscoelastic properties of human arterial walls. Circ Res 18:278292

Matsumoto T, Hayashi K (1996) Stress and strain distribution in hypertensive and normotensive rat aorta considering residual strain. J Biomech Eng 118:62-71. doi: 10.1115/1.2795947

Masson I, Boutouyrie P, Laurent S, Humphrey JD, Zidi M (2008) Characterization of arterial wall mechanical behavior and stresses from human clinical data. J Biomech 41:2618-27. doi: 10.1016/j.jbiomech.2008.06.022

Masson I, Beaussier H, Boutouyrie P, Laurent S, Humphrey JD, Zidi M (2011) Carotid artery mechanical properties and stresses quantified using in vivo data from normotensive and hypertensive humans. Biomech Model Mechanobiol 10:867882. doi: $10.1007 / \mathrm{s} 10237-010-0279-6$ 
Horny L, Netusil M, Vonavkova T (2014) Axial prestretch and circumferential distensibility in biomechanics of abdominal aorta. Biomechanics and Modeling in Mechanobiology 13(4):783-799. DOI: 10.1007/s10237-013-0534-8 MANUSCRIPT http://dx.doi.org/10.1007/s10237-013-0534-8

McEniery CM, Wilkinson IB, Avolio AP (2007) Age, hypertension and arterial function. Clin Exp Pharmacol Physiol 34:665-671. doi: 10.1111/j.1440-1681.2007.04657.x

Ogden RW, Saccomandi G (2007) Introducing mesoscopic information into constitutive equations for arterial walls. Biomech Model Mechanobiol 6:333-344. doi: 10.1007/s10237-006-0064-8

O'Rourke MF, Hashimoto J (2007) Mechanical factors in arterial aging: A clinical perspective. J Am Coll Cardiol 50:1-13. doi: 10.1016/j.jacc.2006.12.050

Rachev A, Greenwald SE (2003) Residual strains in conduit arteries. J Biomech 36:661-70. doi: 10.1016/S00219290(02)00444-X

Schulze-Bauer CAJ, Holzapfel GA (2003) Determination of constitutive equations for human arteries from clinical data. J Biomech 36:165-169. doi: 10.1016/S0021-9290(02)00367-6

Schulze-Bauer CAJ, Morth C, Holzapfel GA (2003) Passive biaxial mechanical response of aged human iliac arteries. J Biomech Eng 125:395-406. doi: 10.1115/1.1574331

Shadwick RE (1999) Mechanical design in arteries. J Exp Biol 202:3305-3313

Sommer G, nad Holzapfel GA (2012) 3D constitutive modeling of the biaxial mechanical response of intact and layerdissected human carotid arteries. J Mech Behav Biomed Mater 5:116-128. doi: 10.1016/j.jmbbm.2011.08.013

Sommer G, Regitnig P, Költringer L, Holzapfel GA (2010) Biaxial mechanical properties of intact and layer-disected human carotid arteries at physiological and supraphysiological loadings. Am J Physiol - Heart Circ Physiol 298:898-912. doi: 10. 1152/ajpheart.00378.2009

Stålhand J (2009) Determination of human arterial wall parameters from clinical data. Biomech Model Mechanobiol 8:141148. doi: 10.1007/s10237-008-0124-3

Stålhand J, Klarbring A (2005) Aorta in vivo parameter identification using an axial force constraint. Biomech Model Mechanobiol 3:191-199. doi: 10.1007/s10237-004-0057-4

Van Loon P, Klip W, Bradley EL (1977) Length-force and volume-pressure relationships of arteries. Biorheology 14:181201

Wilkins K, Campbell NR, Joffres MR, McAlister FA, Nichol M, Quach S, Johansen HL, Tremblay MS. (2010) Blood pressure in canadian adults. Health Reports / Statistics Canada, Canadian Centre for Health Information = Rapports Sur La Santé / Statistique Canada, Centre Canadien d'Information Sur La Santé 21:37-46.

Wittek A, Karatolios K, Bihari P, Schmitz-Rixen T, Moosdorf R, Vogt S, Blase C (2013) In vivo determination of elastic properties of the human aorta based on 4D ultrasound data. J Mech Behav Biomed Mater 27:167-183. doi: 10.1016/j.jmbbm.2013.03.014 